\title{
Numerical study on electrohydrodynamic multiple droplet interactions
}

\author{
P. S. Casas, ${ }^{1}$ M. Garzon,,${ }^{1, *}$ L. J. Gray, ${ }^{2}$ and J. A. Sethian ${ }^{3}$ \\ ${ }^{1}$ Department of Applied Mathematics, University of Oviedo, 33007 Oviedo, Spain \\ ${ }^{2} 119$ Berwick Drive, Oak Ridge, Tennessee 37830, USA \\ ${ }^{3}$ Department of Mathematics, UC Berkeley and Mathematics Department, \\ Lawrence Berkeley National Laboratory, California 94720, USA
}

(Received 18 September 2019; published 30 December 2019)

\begin{abstract}
We present a numerical study of inviscid multiple droplet coalescence and break-up under the action of electric forces. Using an embedded potential flow model for the droplet hydrodynamics, coupled with an unbounded exterior electrostatic problem, we are able to perform computations through various singular events and analyze the effects of the electrical field intensity on droplet interactions. Laboratory experiments on the electrodynamics of droplet pairs show a much richer, and sometimes unexpected, behavior than that of isolated droplets. For example, it has been found that opposite charged droplets tend to repel each other when the electric field intensity is above a certain critical value. Although the mathematical model employed in this work incorporates very simple flow and electric assumptions, many of the droplet coalescence patterns seen in laboratory experiments can be reproduced. In this model, the interaction pattern of two droplets of radii $R_{0}$ separated a distance $D_{0}$, depends on the ratio $X_{0}=D_{0} / R_{0}$ and the applied uniform electric field intensity, $E_{\infty}$. By performing a vast number of numerical simulations we are able to characterize the coalescence modes before and after drop merging as a function of these two parameters. The simulations predict that droplet repulsion occurs within a narrow interval of $E_{\infty}$ values, different for each $X_{0}$. Surprisingly, in this $E_{\infty}$ interval, a sharp transition between two power-law precoalescence flow regimes is seen. The evolution of several flow characteristics before and after coalescence, and the shape of the deformed droplets at coalescing time and the double cone angle, are also addressed and analyzed in detail. Cone angles below $35^{\circ}$ lead to droplet coalescence for any $X_{0}$ value, which is in accordance with previously reported studies. Finally, it is shown that the model and algorithm can handle multiple droplet interactions. The simulations qualitatively match results from water in oil experiments in microchannels, despite the fact that the exterior fluid is not considered in the mathematical model.
\end{abstract}

DOI: 10.1103/PhysRevE.100.063111

\section{MOTIVATION AND OVERVIEW}

Electric forces acting on droplet pairs or multiple drop flows trigger richer dynamics than those provoked on isolated droplets. The effectiveness of industrial processes, such emulsion evolution, droplet-based microfluidic devices, rain formation in clouds, industrial spray painting, etc., depends on fully understanding these phenomena.

When a neutral and isolated droplet is exposed to a uniform electric field in the direction of the droplet symmetry axis, it will oscillate with a frequency that depends on the electric field intensity. Taylor [1] found a theoretical critical value above which the drop will distort and elongate until tip streaming will take place from the well-known Taylor cones. Numerous experimental [2,3] and computational studies [4-7] have corroborated these dynamics. Inviscid coalescence of touching droplet pairs, of either the same or different sizes, has also been a subject of intense research, see, for example, Refs. [8,9] for results of laboratory experiments and Refs. [10-12] for numerical simulations. Under this configuration the merging mechanism is due to surface tension and the system seeks to minimize surface energy. A thin connecting bridge is initially formed and its evolution seems

*maria.garzon.martin@gmail.com to follow a self-similar power law. Capillary waves propagate along the drop surface, causing the drop go from elongated to capsulelike shapes until equilibrium is achieved. The coalescence of unequal size drops, however, leads to pinch-off of a satellite droplet whose size depends on the relative sizes of the parent droplets $[9,12]$.

In some applications, e.g., removing water droplets from crude oil, it is necessary to bring the droplets together prior to coalescence. The use of electrical forces is a well-known technique to make drops attract each other and merge. Most of the existing studies start with a pair of droplets of radii $R_{0}$ immersed in a dielectric medium, separated by a distance $D_{0}$, and exposed to a direct current (dc) or an alternating current (ac). The system can be open or confined in a microchannel and the electrical properties of the liquid droplets and surrounding medium can also vary. Studies of the dynamics of droplet pairs aligned with an electric field can be found in Refs. [13-15] for viscous fluids and in Ref. [16] for inviscid fluids. In these scenarios the droplet pair acts as a dipole enhancing the action of the applied electric field at the drop ends $[17,18]$ and therefore the critical electric intensity to destabilize the drops diminishes.

Recent laboratory experiments have found an unexpected droplet pairs electrical interaction. Above a certain value of the electric field strength, it has been observed [19] that opposite charged water droplets dispersed in oil repel each 

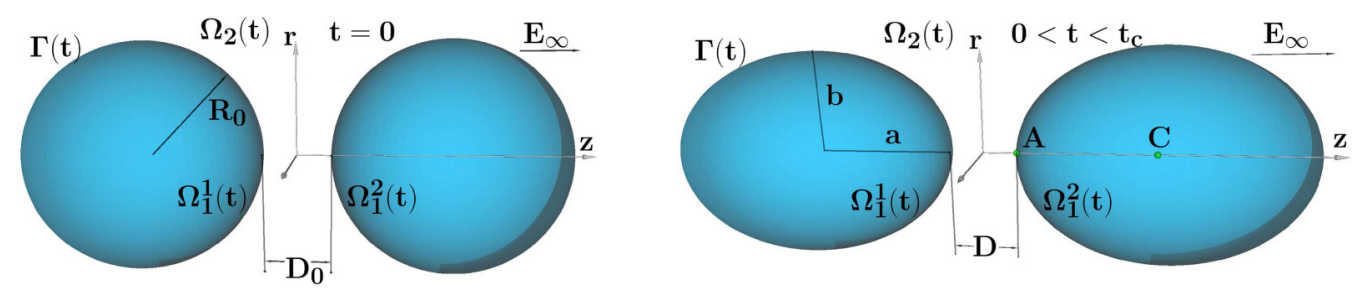

FIG. 1. Physical sketch of the droplet pair geometry. Left: Two spherical droplets of radius $R_{0}$ at initial time. Right: The deformed droplets with aspect ratio $a / b$. Points on the right droplet, $\left(0, z_{C}\right)$ the droplet center, and $\left(0, z_{A}\right)$ the droplet left end point, are depicted.

other after they first contact. Bird et al. [20] also observed the same phenomena for two water drops suspended on needles. At a low voltage difference between the needles, the facing drop ends will deform into cone shapes with angle $\beta$ and immediately coalesce (see Fig. 1 in Ref. [20] for the angle definition). For certain values of the voltage difference the drops will touch but then recoil preventing coalescence. The coalescence stability diagram for droplet pairs on microfluid devices has been experimentally established in Ref. [21]; these authors also report interesting experimental results on multiple drop interaction. A complete numerical study on drop pair interactions in a microchannel has been reported in Ref. [15]. Finally, Chabert et al. [22] assert that a dc current cannot induce coalescence in a microchannel, and thus they imposed an ac current and leaky dielectric assumptions for both liquid phases. Nevertheless, despite all these important experimental, theoretical, and numerical studies, there still remains controversy about the mechanisms and conditions that lead to recoil after two drops make contact under the action of electric forces. From the above-mentioned works, geometric features at initial contact time, such as the double cone angle $\beta$, seem to play a definite role.

We believe that drop pair studies, under basic flow and electric assumptions that involve few parameters, can contribute to the understanding of this phenomena. Therefore, herein we focus on the electrodynamics of a nonviscous droplet pair inside a dynamically inactive exterior fluid, subject to a uniform electric field (dc current). Gravity forces are considered to be negligible compared to surface tension forces within the droplet length scales reported in experiments. A pioneer work with these same assumptions was conducted by Adamiak [16], but his numerical approximation give rise to numerical instabilities for some parameter values and no computations after coalescence were possible. However, with the level set or extension potential methods employed herein, the computations can go past singular events, such as drops breaking or merging. The reliance of our model formulation on the level set techniques enables moving boundary equations to be transformed into partial differential equations (one dimension higher) posed on a fixed domain. The resulting system of partial differential equations is approximated combining upwind finite differences schemes for the time-dependent embedded equations and linear boundary elements for the integral formulation of the Laplace equations. This approach has been used to solve various hydrodynamical problems, such as wave breaking [23], the Taylor-Rayleigh instability of a fluid jet, [24], and droplet and bubble evolution in a two-fluid system $[25,26]$. Regarding electrohydrodynamical processes, droplet deformation and jetting in axisymmetric geometries are reported in Ref. [7] and preliminary results on electrocoalescence also appear in Ref. [12]. In fact, the present work can be regarded as a natural continuation of Ref. [12]. Although we have also developed a complete three-dimensional (3D) algorithm for electrohydrodynamical problems (based on the coupling of level set method with Nitche finite element method, see Ref. [27]), the computational times needed to carry out 3D pair-drops and multiple-drop simulations exceed our capabilities, and the axisymmetric assumption will be again employed.

The most important findings extracted from the wide range of numerical simulations carried out in this work are as follows:

(i) Droplet coalescence and droplet repulsion are captured by this model and, consequently, these simulations can help to understand the underlying physics of this phenomenon. Precoalescence flow analyses show the existence of two regimes: In the first regime electric and surface tension forces alternate, whereas in the second regime electric forces prevail. It is in the transition between these regimes where droplet repulsion takes place. It appears that droplet recoiling is not only caused by local geometric features at the contact point but also by a combination of local and global dynamics.

(ii) Electric postcoalescence has also been investigated. We have identified four distinct patterns of postcoalescence evolution in the presence of an electric field: stable oscillation, satellite droplets formation, droplets repulsion, and droplet destabilization with jet emission. By inspecting the computational results the possible mechanisms for each of these postcoalescence modes have been proposed.

(iii) Multiple drop stable coalescence depends on the initial droplet separation and electric field intensity. Available laboratory experiments on droplet trains use water droplets dispersed in an oil phase. We are aware that our model does not incorporate the viscosity effects on the free surface due to the exterior fluid. Nevertheless, the numerical computations give reasonable qualitative agreement when compared to experimental results, qualifying this model as a predictive tool for multiphase separation studies.

This paper is organized as follows: Section II briefly presents the mathematical model and approximation techniques, and further details can be found in Ref. [12]. Section III is devoted to the numerical simulations and analysis of droplet pairs electrohydrodynamics. We consider droplet pairs located at different initial rescaled distance, $X_{0}=\frac{D_{0}}{R_{0}}$, exposed to various electric field intensities, $E_{\infty}$. From this study the coalescence modes in $\left(X_{0}, E_{\infty}\right)$ space and the main flow characteristics prior and post coalescence can be established. Cone angles and droplet shapes at contact 
time are also addressed and compared to previously reported values. A mechanism for droplet repulsion is proposed and a comparison with laboratory experiments is also addressed. Finally, Sec. IV considers the action of a uniform electric field on aligned droplets of equal radii located at equal or unequal initial distances. The numerical results are compared with laboratory experiments.

\section{ELECTROHYDRODYNAMIC MODEL AND NUMERICAL APPROXIMATION}

The physical setting to be modeled is the interaction of two or, more generally, $M$ droplets of a perfect conductor fluid immersed in a perfect dielectric medium. The drop centers are aligned parallel with the electrical far field direction. The flow inside the drops is considered inviscid and the medium is dynamically at rest. Each drop occupies a domain $\Omega_{1}^{k}$ with boundary $\Gamma_{k}(t), \quad k=1, \ldots, M$, whereas the infinite dielectric medium fills the $\Omega_{2}(t)$ domain. The physical parameters involved are the drops fluid density $\rho$, the surface tension coefficient $\gamma$, and the insulator gas (or liquid) permittivity constant $\epsilon$.

Let be $\Omega_{1}(t)=\cup_{k=1}^{M} \Omega_{1}^{k}$ and $\Gamma(t)=\cup_{k=1}^{M} \Gamma_{k}(t)$. Taking as characteristic length scale $R_{0}$, the droplets radii, capillary timescale $t_{0}=\left(\rho R_{0}^{3} / \gamma\right)^{1 / 2}$, and electric field intensity scale $E_{0}=\left[2 \gamma /\left(\epsilon R_{0}\right)\right]^{1 / 2}$, the nondimensional fluid flow Lagrangian equations are as follows:

$$
\begin{array}{ll}
\mathbf{u}=\nabla \phi & \text { in } \Omega_{1}(t), \\
\Delta \phi=0 & \text { in } \Omega_{1}(t), \\
\frac{\partial \mathbf{R}}{\partial t}=\mathbf{u} & \text { on } \Gamma(t), \\
D_{t} \phi=f & \text { on } \Gamma(t),
\end{array}
$$

where $\mathbf{R}(x, y, z, t)$ is the position vector of a fluid particle on the front, $\mathbf{u}=\mathbf{u}(x, y, z, t)$ is the velocity field, $\phi=$ $\phi(x, y, z, t)$ the velocity potential, and $D_{t}$ stands for the convective derivative, $D_{t} \phi=\frac{\partial \phi}{\partial t}+\mathbf{u} \cdot \nabla \phi$. The function $f=$ $f(x, y, z, t)$ contains the contribution of the various forces acting on $\Gamma(t)$.

In the unbounded exterior domain $\Omega_{2}(t)$ we set $\mathbf{E}$ to be uniform and parallel to the symmetry axis at the far field. Under these assumptions $\mathbf{E}$ is solenoidal and can be expressed as the gradient of an electric potential field $U=U(x, y, z, t)$ :

$$
\begin{gathered}
\mathbf{E}=-\nabla U \quad \text { in } \Omega_{2}(t), \\
\Delta U=0 \quad \text { in } \Omega_{2}(t), \\
U=U_{0}^{k}(t) \quad \text { on } \Gamma_{k}(t), \quad k=1, \ldots, M, \\
U=-E_{\infty} z \quad \text { at infinity. }
\end{gathered}
$$

Here $E_{\infty}$ is the nondimensional electric field intensity at the far field, and under these assumptions the flow is driven by inertia, surface tension, and electric stresses. The function $f$ in Eq. (4) is then

$$
f=\frac{1}{2}|\mathbf{u}|^{2}-\kappa+E_{n}^{2}
$$

where $\kappa$ is twice the mean curvature, $E_{n}=\mathbf{n} \cdot \nabla U$, and $\mathbf{n}$ the unit normal vector pointing from the interior to the exterior fluid domain. We point out that the free boundary electric potentials $U_{0}^{k}(t), k=1, \ldots, M$ are unknown, and as the $M$ drops interact, these potentials cannot be obtained independently. The algorithm for obtaining the potentials, for either neutral or charged droplets, can be found in Ref. [12].

Note that Eqs. (3) and (4), the kinematic and dynamic boundary conditions on the moving domain boundary, $\Gamma(t)$, are two surface partial differential equations (PDE's) that define the evolution of the free boundary and the associated velocity potential within this boundary. The numerical approximation of these two equations is what makes this problem very challenging, especially if $\Gamma(t)$ changes topology. Equation (4) is the conservation of momentum on the free boundary, where the continuity of the stress tensor has been imposed and thus the coupling term between the interior and exterior problems is set by Eq. (9). It is, in theory, possible to approximate the coupled system of PDE's using a front tracking method for Eqs. (3) and (4), choosing $P_{k}$ fluid particles in each free front $\Gamma_{k}$ and solving a system of $2 \times P_{k}$ ordinary differential equations for each front. For multiple droplets exhibiting merging and breaking processes this approach is almost impossible, as it is difficult to assign the marker particles to the appropriate volumes.

The alternative to this approach is to reformulate the surface equations (3) and (4), posed on $\Gamma(t)$, as one-higher dimension equations posed on a fixed domain $\Omega_{D}$, chosen as a rectangular domain that should contain $\Gamma(t)$ for all computational times. Other than this requirement, there is no relation with the physical domains. The embedding of (3) and (4) on $\Gamma(t)$ onto $\Omega_{D}$ is accomplished with level set techniques. Define two new functions in $\Omega_{D}$, the level set function $\Psi(x, y, z, t)$ and the extended potential function $G(x, y, z, t)$, via

$$
\begin{gathered}
\Psi(\mathbf{R}(\mathbf{s}, t), t)=0, \quad \forall t \in[0, T], \\
\left.G(\mathbf{s}, t)\right|_{\Gamma(t)}=\phi(x, y, x, t) \forall t \in[0, T],
\end{gathered}
$$

where $\mathbf{s}=\left(s_{1}, s_{2}\right)$ an arc length parametrization of the free boundary surface. Equations (3) and (4) transform into

$$
\begin{gathered}
\Psi_{t}+\mathbf{u}_{\mathrm{ext}} \cdot \nabla \Psi=0 \text { in } \Omega_{D}, \\
G_{t}+\mathbf{u}_{\mathrm{ext}} \cdot \nabla G=f_{\mathrm{ext}} \text { in } \Omega_{D},
\end{gathered}
$$

and $\mathbf{u}_{\text {ext }}$ and $f_{\text {ext }}$ denote the extended velocity and extended function $f$ from $\Gamma(t)$ onto $\Omega_{D}$. The model equations for electrohydrodynamic problems using the level set embedding are thus:

$$
\begin{gathered}
\mathbf{u}=-\nabla \phi \quad \text { in } \Omega_{1}(t), \\
\Delta \phi=0 \quad \text { in } \Omega_{1}(t), \\
\phi=G \quad \text { on } \Gamma(t), \\
\Psi_{t}+\mathbf{u}_{\mathrm{ext}} \cdot \nabla \Psi=0 \quad \text { in } \Omega_{D}, \\
G_{t}+\mathbf{u}_{\mathrm{ext}} \cdot \nabla G=f_{\mathrm{ext}} \text { in } \Omega_{D},
\end{gathered}
$$




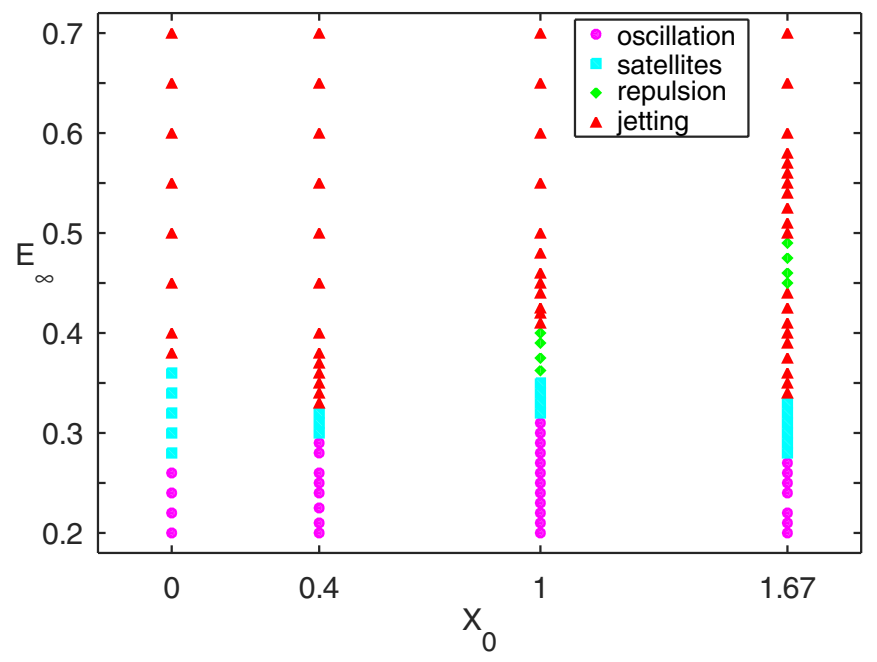

FIG. 2. Coalescence modes on $\left(X_{0}, E_{\infty}\right)$ space. Each point on the graph corresponds to a numerical simulation. For $X_{0}=$ $0,0.4,1,1.67$ the postcoalescence sequence for increasing values of $E_{\infty}$ is oscillation, satellites, and jetting. The repulsion mode appears for $X_{0}=1$ and $X_{0}=1.67$ at different $E_{\infty}$ intervals.

$$
\begin{gathered}
\mathbf{E}=-\nabla U \quad \text { in } \Omega_{2}(t), \\
\Delta U=0 \quad \text { in } \Omega_{2}(t), \\
U=U_{0}^{k}(t) \quad \text { on } \Gamma_{k}(t), \quad k=1, \ldots, M, \\
U=-E_{\infty} z \quad \text { at infinity. }
\end{gathered}
$$

The boundary conditions for (17) and (18) can be set to $\mathbf{n}_{D} \cdot \nabla \Psi=0$ and $\mathbf{n}_{D} \cdot \nabla G=0$, respectively, on $\partial \Omega_{D}$, with $\mathbf{n}_{D}$ denoting the exterior normal to $\partial \Omega_{D}$. These assumptions mean that the level sets of $\Psi$ and $G$ will intersect $\partial \Omega_{D}$ orthogonally.

The numerical approximation can be described in three steps:

(i) The time discretization for Eqs. (17) and (18) is approximated using a first-order explicit Euler scheme or second-order Runge-Kutta (the latter requires much more computational time and improved accuracy is only detected on analytic test problems).

(ii) The spatial discretization of the gradient terms, $\nabla \Psi^{n}$, $\nabla G^{n}$, at each time step employs a first- or second-order upwind scheme on a rectangular uniform mesh over $\Omega_{D}$.

(iii) At each discrete time, $t^{n}$, the interior and exterior Laplace equations are solved using a linear element Galerkin boundary integral formulation. Details concerning the axisymmetric Galerkin implementation can be found in Ref. [28].

The axisymmetric assumptions lead to a problem posed on the $(r, z)$ plane, with $z$ as the symmetry axis. There are therefore two meshes involved in the algorithm: a rectangular uniform grid over $\Omega_{D}$ with mesh size $\Delta r=\Delta z$ and a mesh of size $d s$ to discretize the free boundary $\Gamma(t)$. This length $d s$ can be uniform or nonuniform depending on the resolution needed at various regions. The adaptive time step, $\Delta t$, is chosen according to formula 31 in Ref. [12]. Further details on the numerical approximation and algorithm can be found in Refs. [7,12,24,26,27,29,30]. In particular, we note that an exhaustive convergence analysis with respect discretization parameters can be found in Ref. [24]. In this analysis the short-time analytic solution for the Rayleigh-Taylor instability problem was employed. Moreover, break-up similarity scaling laws and postbreaking events were seen to be independent of discretization parameters, and conservation of mass and energy held through these singular events. In Ref. [25] the analytic solution to the problem of a perturbed spherical shape droplet also demonstrated convergence, and in Ref. [12] the neck expansion after coalescence of two equal tangentially touching droplets was seen to converge with diminishing mesh sizes and time steps.

\section{DROPLET PAIR ELECTROHYDRODYNAMICS}

We first consider two neutral spherical droplets of radius $R_{0}$ separated by a distance $D_{0}$ and set in motion by a uniform electric field of intensity $E_{\infty}$, see Fig. 1 for a schematic view. The coalescence time, the shape of the end point regions and the double cone angle at contact time all vary with these parameters. By performing a large number of

TABLE I. Three dimensional renderings of the droplets profiles showing oscillation, satellites, repulsion, and jetting postcoalescence modes.

\begin{tabular}{|c|c|c|c|c|}
\hline \hline Oscillation & satellites & repulsion & jetting \\
\hline & & & & \\
\hline
\end{tabular}


numerical simulations we have been able to study not only the flow characteristics prior to merging but also the complex postcoalescence evolution.

In particular, two important aspects of the droplet pair dynamics will be explored:

(1) Droplets coalescence versus droplets repulsion after first contact.

(2) Possible mechanisms underlying the various postcoalescence modes.

Figure 2 and Table I provide a useful visualization of the droplet pair behavior depending on the $\left(X_{0}, E_{\infty}\right)$ space and movies corresponding to these four patterns can be seen in Supplemental Material [31]. In this figure, four coalescence modes have been identified:

(i) Droplets merge and oscillate. This mode is represented by magenta circles.

(ii) Droplets coalesce and the merged droplet elongates sufficiently that two satellite droplets carrying opposite charges pinch-off (represented by cyan squares).

(iii) Droplets touch each other but immediately recoil and fail to coalesce (represented by green diamonds).

(iv) Droplets coalesce, the merged droplet elongates, destabilizes and jet ejection occurs (represented by red triangles).

Figure 2 represents a total of 104 simulations with a maximum computational elapsed time of $T=4$ nondimensional units. The fixed domain $\Omega_{D}$ that contains the droplets for all times is $\Omega_{D}=[-3,3] \times[-1,1]$. From previous experiments and convergence test we have found that a workable set of discretization parameters is as follows: $\Delta r=\Delta z=0.005$, $d s \in[0.01,0.02]$, and $\Delta t=10^{-3}$ initially and going down to $10^{-5}$ near singular events.

To establish the domains of the different droplet evolution, characterized by the final droplet fate after first contact, a detailed investigation of the influence of $E_{\infty}$ is needed for each $X_{0}$. We have selected $X_{0}=0,0.4,1,1.67$, and $E_{\infty}$ is taken in the interval $[0.2,0.7]$ incremented 0.02 units and, if needed, by 0.01 units. The lower bound has been set to $E_{\infty}=0.2$, as below this value and for $X_{0}=1.67$ the droplets will not coalesce in $t \in[0, T]$, whereas above 0.7 the post coalescence leads immediately to jet ejection. Moreover, for $X_{0}>1.67$, the computation time needed for the droplets to contact is excessive.

We first describe and analyze droplet geometric features and observable kinematics considering three separate stages: precoalescence evolution, contact characteristics, and postcoalescence dynamics. We then close this section with a discussion of the mechanism behind repulsion versus coalescence and a comparison with existing laboratory experiments.

\section{A. Electrodynamics of approaching drops}

In order to characterize this initial stage, the evolution of various geometric parameters is tracked. In particular, the droplets aspect ratio, $a / b$, the droplet center axial coordinate, $z_{C}$, and the droplet left end point coordinate, $z_{A}$, are studied, see Fig. 1 for a depiction of these quantities. The coalescence times, $t_{c}$, are also analyzed. Figure 3 shows the aspect ratio versus time for $X_{0}=0.4,1,1.67$. It can be observed that for low values of $E_{\infty}$ and $X_{0}=1,1.67$ the drops oscillate as
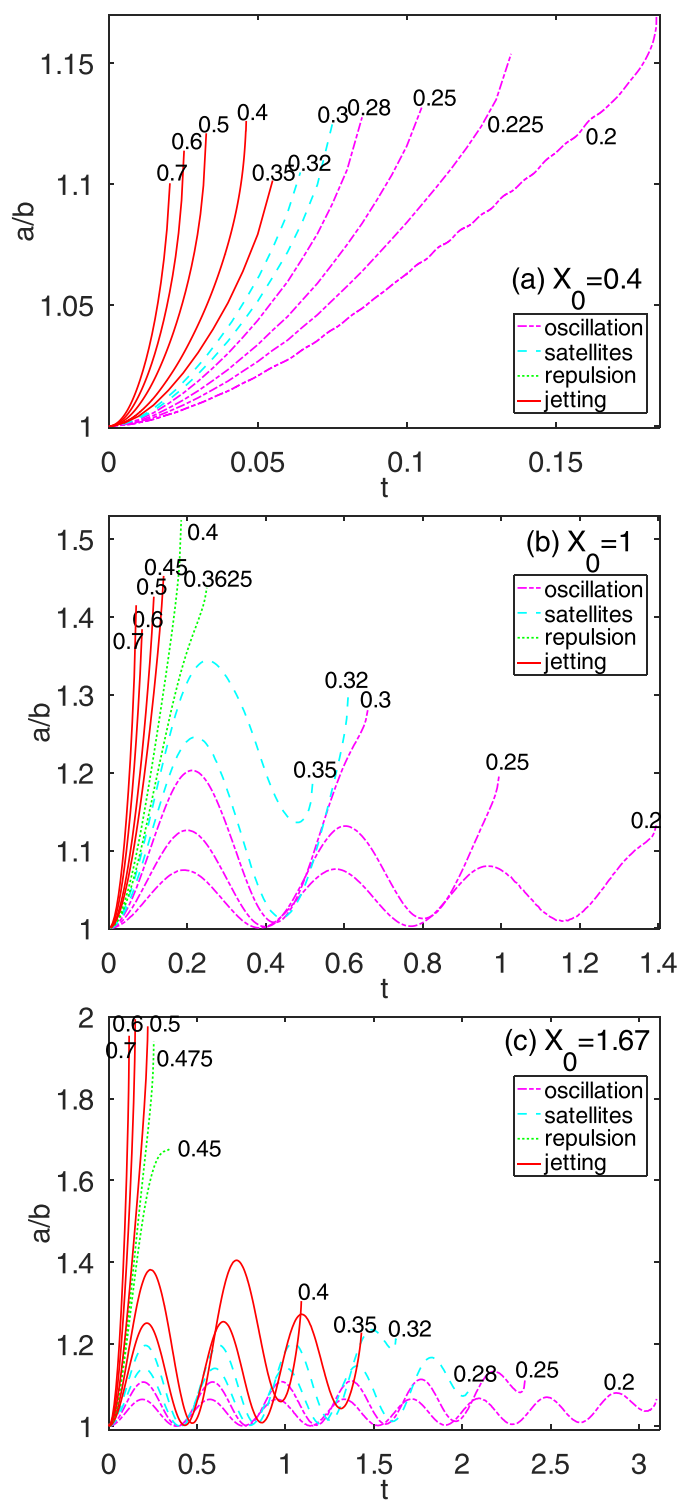

FIG. 3. Evolution of the droplets aspect ratio $a / b$ for various electric fields intensities $E_{\infty}$. From top to bottom the graphs correspond to $X_{0}=0,1,1.67$, respectively. For $X_{0}=0.4$ the precoalesced droplets do not oscillate. The repulsion mode appears between precoalescence oscillation and precoalescence pure deformation. The curve colors and line styles represent the corresponding postcoalescence modes.

they approach each other with an amplitude that increases with the electric field intensity. For a particular $E_{\infty}$ the amplitude also increases as the droplet distance diminishes, indicating the time when the opposite charges on the facing droplet boundaries start to affect the distortion. However, for $X_{0}=0.4$ there is no oscillation and the droplets deform with aspect ratios $a / b<1.2$ for all values of $E_{\infty}$. Note that the assigned curve colors are consistent with those employed to identify the postcoalescence modes in $\left(X_{0}, E_{\infty}\right)$ space: This hopefully helps in the understanding of the computed results.

For the rescaled distance $X_{0}=1$, droplet aspect ratios below 1.3 lead to the merged droplet oscillation mode, whereas for $a / b \in[1.4,1.5]$, repulsion or jetting will occur. In the 

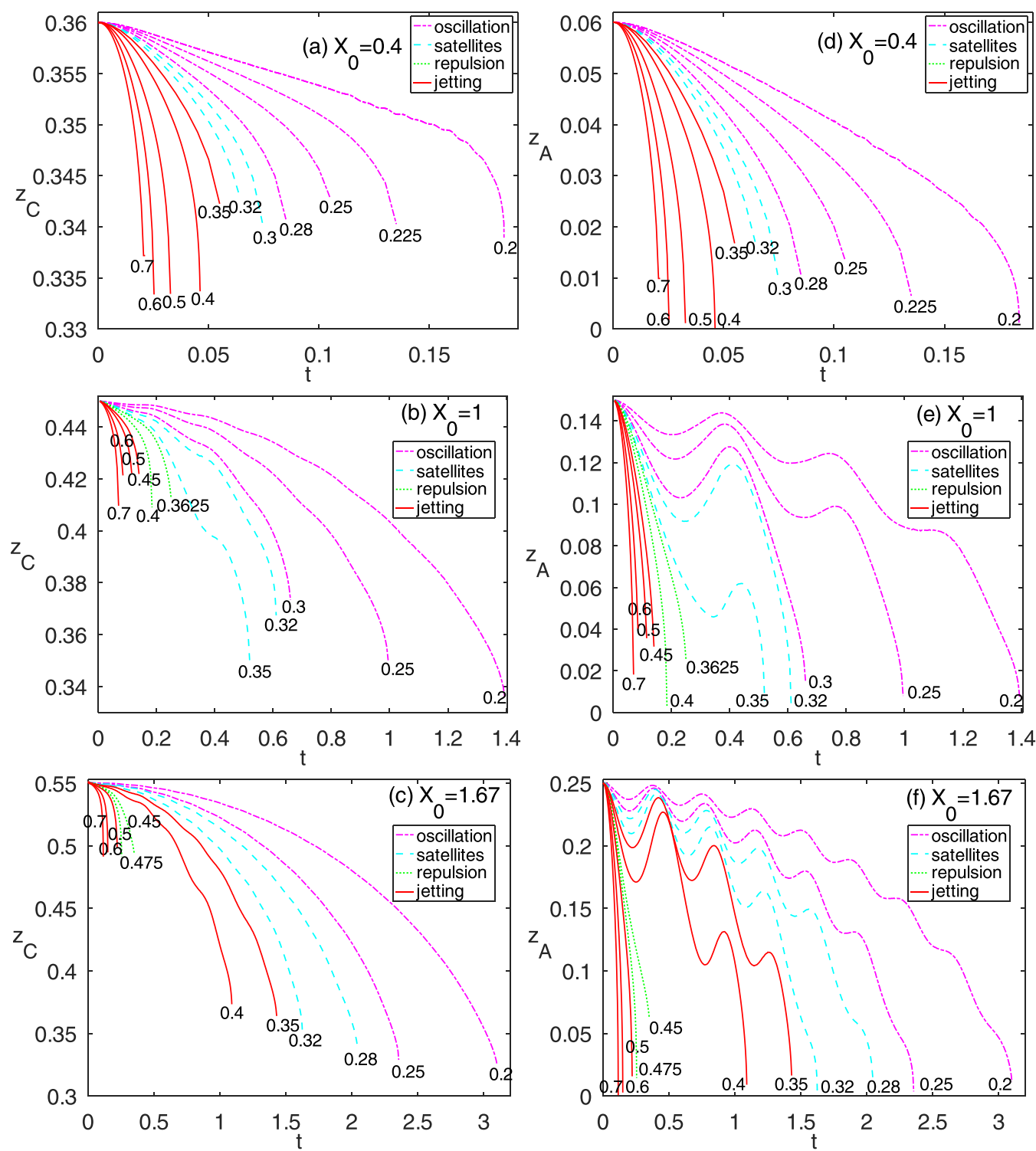

FIG. 4. Left: Evolution of the droplets center axial coordinate $z_{C}$ for various electric field intensities and indicated $X_{0}$ values. Right: Same for the evolution of the drop left point $z_{A}$. The curves color and line styles represent the corresponding postcoalescence modes.

interval $1.3<a / b<1.4$ the coalescence satellite mode will take place. Regarding $X_{0}=1.67$ the dynamics of the aspect ratio is more complex: We detected oscillation, satellite, and jetting coalescence modes for aspect ratios below 1.4, which corresponds to values of $E_{\infty}<0.4$, whereas repulsion or jetting mode appears for $a / b>1.4$. Note that the repulsion mode shows up in a short range of $E_{\infty}$ values and just in the border of precoalescence oscillation and pure deformation. For values of $E_{\infty}>0.4$ the drops do not oscillate but just translate as they deform reaching aspect ratios $a / b \approx 2$ at coalescence.

On the left in Fig. 4 the evolution of the axial coordinate of the drop center, $z_{C}$, is depicted, while the right column shows the end point, $z_{A}$. While the evolution of $a / b$ gives a geometrical characteristic of the droplet shape, the analysis of $z_{C}$ and $z_{A}$ reveals kinematic aspects of the movement. It can be observed that the drop centers move steadily toward each other for all $X_{0}$ values, whereas for $X_{0}=1.0$ and $X_{0}=1.67$ the drop end points oscillate while approaching coalescence. The velocity of the drop centers for each value of $E_{\infty}$ is not constant but rather increases as the distance between the droplet pair decreases; similarly, the amplitude of the oscillations in $z_{A}$ increases with $E_{\infty}$. At first glance $z_{C}$ seems to behave as quadratic functions of time but attempts to fit to a parabola were not successful. The drop translation is therefore not a uniform rectilinear motion.

Finally Fig. 5 (on the left) displays the coalescence time, $t_{c}$, plotted versus electrical field intensity for the three $X_{0}$ values; the corresponding log-log plots are on the right. The logarithmic graphs reveal a sharp change between two different regimes, with both regimes following power laws with similar exponents for all $X_{0}$ values. The jump between the regimes occurs at different $E_{\infty}$ values for each $X_{0}$. For $X_{0}=1.67$ the transition occurs within $E_{\infty} \in[0.4,0.5]$, and in the interval $E_{\infty} \in[0.35,0.4]$ for $X_{0}=1$. The $X_{0}=0.4$ plot is mostly on the second regime, with a smother transition 

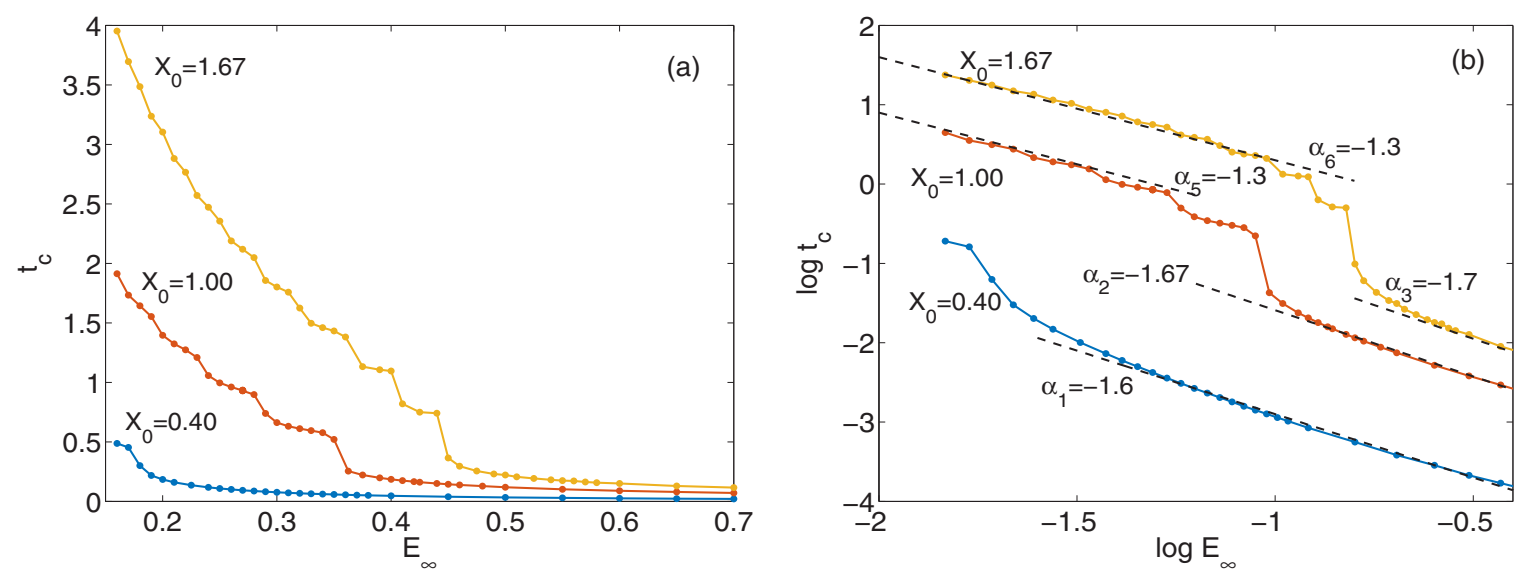

FIG. 5. Left: Coalescence times $t_{c}$ versus $E_{\infty}$. Right: Corresponding log-log plot with the exponents of the linear fittings. The log is natural logarithm. For $X_{0}=1,1.67$ there is a curve jump between the two distinct flow regimes.

within $E_{\infty} \in[0.25,0.29]$. The Matlab linear fit has given the following power-law exponents for the second regime: $\alpha_{1}=$ $-1.6, \alpha_{2}=-1.67$ and $\alpha_{3}=-1.7$ for $X_{0}=0.4,1,1.67$, respectively. The exponents for the first regime are $\alpha_{5}=\alpha_{6}=$ -1.3 for $X_{0}=1,1.67$. There is not enough data to fit a straight line to the first regime in the $X_{0}=0.4$ plot.

Next we investigate the double cone angle $\beta$ and other droplet features at coalescence.

\section{B. Contact events: Double cone angle and end-point shape}

It has been observed that when a pair of droplets make contact the near faces adopt a shape that can be inscribed into a double cone with an opening angle $\beta$, see Fig. 8. Recent experimental observations $[19,20]$ show that, depending on the angle $\beta$, opposite charged droplets repel each other after first contact. Ristenpart et al. [19] gave a physical explanation involving the pressure difference between the bulk of the drop and the meniscus bridge. However, this leads to a critical angle of $\beta=45^{\circ}$, while their laboratory experiments show an angle of $\beta=31^{\circ}$. A local theoretical model in Ref. [20], based on the minimization of an energy functional for the neck region, gives a critical angle of $\beta=30.8^{\circ}$. The experiments set reported in Ref. [20] found that neither the dielectric strength of the surrounding gas or the conductivity of the drops liquid were responsible of the repulsion, concluding that it is solely a consequence of the geometric shape at contact time. For a constant electric field across two inviscid drops,

TABLE II. Electric field intensity, $E_{\infty}$, coalescence time, $t_{c}$, and cone angle, $\beta$, for $X_{0}=0.4,1,1.67$.

\begin{tabular}{|c|c|c|c|c|c|c|}
\hline \multirow[b]{2}{*}{$E_{\infty}$} & \multicolumn{2}{|c|}{$X_{0}=0.4$} & \multicolumn{2}{|c|}{$X_{0}=1.0$} & \multicolumn{2}{|c|}{$X_{0}=1.67$} \\
\hline & $t_{c}$ & $\beta$ & $t_{c}$ & $\beta$ & $t_{c}$ & $\beta$ \\
\hline 0.2 & 0.1838 & 24.0 & 1.3960 & 27.0 & 3.1035 & 27.8 \\
\hline 0.3 & 0.0761 & 31.0 & 0.6624 & 32.6 & 1.8016 & 30.1 \\
\hline 0.4 & 0.0462 & 30.1 & 0.1849 & 39.0 & 1.0955 & 34.8 \\
\hline 0.5 & 0.0328 & 32.8 & 0.1191 & 41.0 & 0.2218 & 47.2 \\
\hline 0.6 & 0.0254 & 32.9 & 0.0890 & 44.7 & 0.1501 & 51.0 \\
\hline 0.7 & 0.0210 & 37.5 & 0.0709 & 45.3 & 0.1156 & 52.5 \\
\hline
\end{tabular}

Ref. [18] gives the value $\beta=40.1^{\circ}$, whereas Ref. [15] reports $\beta=45^{\circ}$ for droplet pairs in microchannels.

We have calculated the $\beta$ angle corresponding to $X_{0}=$ $0.4,1,1.67$ and various $E_{\infty}$ values by performing a linear fit to front coordinates $(r, z)$ near the contact point $(0,0)$. Table II lists the cone angles $\beta$ together with the coalescence times $t_{c}$. It can be seen that there is a sharp change in the trend of $t_{c}$ and $\beta$ within certain $E_{\infty}$ intervals. Figure 6 plots $\beta$ versus $E_{\infty}$ for the three $X_{0}$ values. It is very interesting to observe that $\beta$ undergoes a trend change at same $E_{\infty}$ intervals as the coalescence time (Fig. 5). Not surprisingly, inspecting Fig. 2, the recoil coalescence mode occurs precisely within these same electric intensity ranges for each of the $X_{0}$ values. According to these numerical results, the limiting cone angle for coalescence depends on the $X_{0}$ value. For $X_{0}=1.67$, within the interval $[0.45,0.5)$ the droplet pair fail to merge and therefore $\beta \leqslant 38.9^{\circ}$ will lead to coalescence. The recoil region for $X_{0}=1$ occurs at $E_{\infty} \in[0.35,0.40]$ and the limiting angle is now $\beta \leqslant 35^{\circ}$. Therefore for cone angles below $\beta=35^{\circ}$ the droplets coalesce. Although these

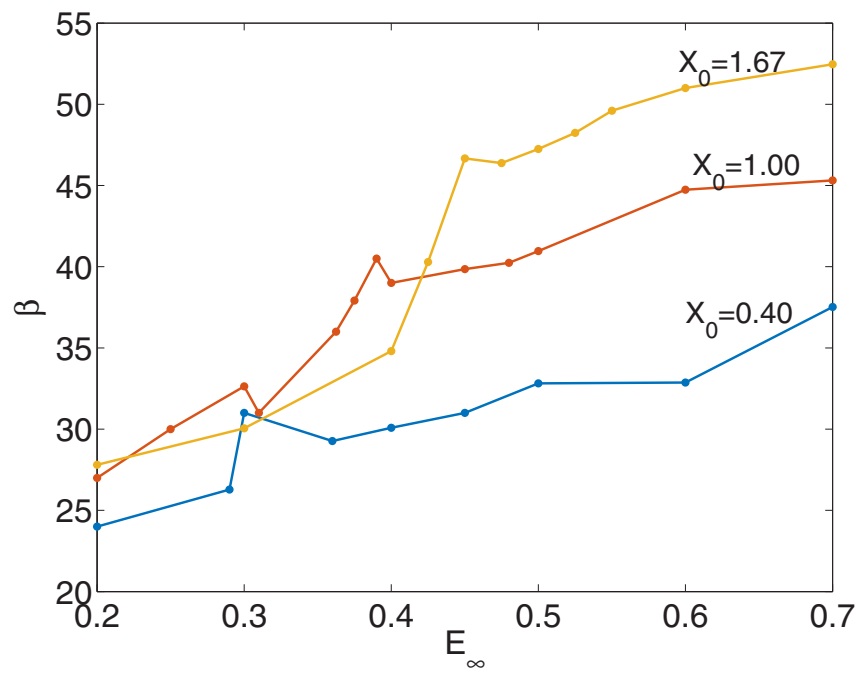

FIG. 6. Double cone angle $\beta$ versus $E_{\infty}$, for $X_{0}=0.4,1,1.67$. 


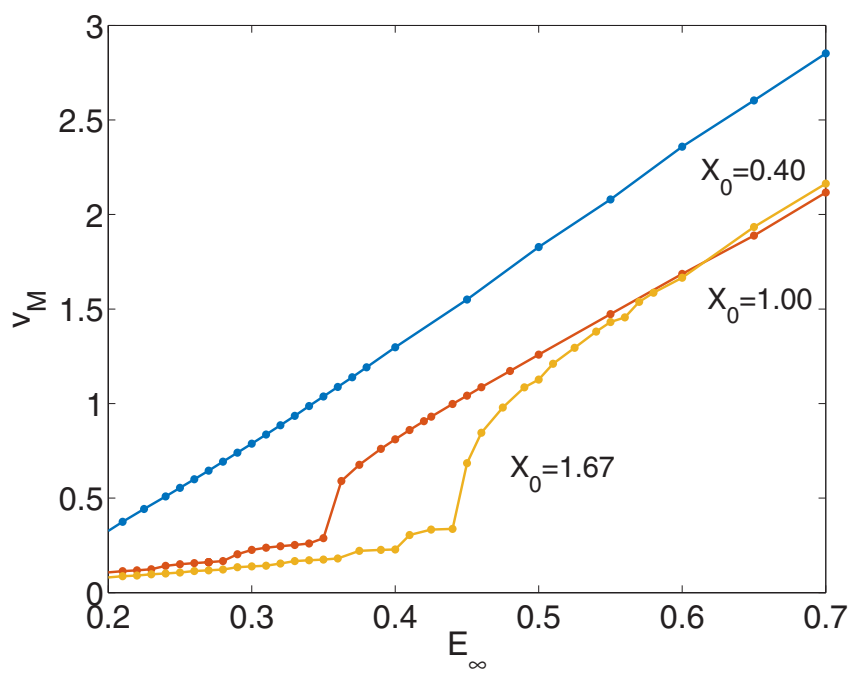

FIG. 7. Average droplet velocity $V_{M}=D_{0} / t_{c}$ versus $E_{\infty}$ for $X_{0}=$ $0.4,1,1.67$. For $X_{0}=1,1.67$ the average velocity is $V_{M} \approx 0.5$ at the curve jumps.

values are in good agreement with local theoretical models, previous numerical simulations, and experiments, the recoiling coalescence mode seems to depend on more than just geometric features (such as the curvature inside and across the thin bridge formed at contact). The velocity at which the droplets make contact should be a factor, and to investigate this Fig. 7 shows the average velocity $V_{M}=D_{0} / t_{c}$ versus $E_{\infty}$. It can be seen that at precisely the critical $E_{\infty}$ intervals, the average droplet velocity is almost the same for all three $X_{0}$ values.

We note that low $E_{\infty}$ values lead to a clear double cone angle contact profile, and this allows a robust and consistent calculation of $\beta$. For high $E_{\infty}$ values, however, the local electrophoretic forces deform the drops tips sufficiently that the calculation of the cone angle is subject to a variation of one or two degrees, depending on the fitted region. Figure 8 depicts the fronts and the fitted cone angles for $X_{0}=1.67$ and $E_{\infty}=0.2,0.4,0.6$. Same procedure has been used to obtain all reported $\beta$ angles.

We now discuss the droplet facing profiles near contact time, taking $X_{0}=1.67$. Values in the range $0.2 \leqslant E_{\infty} \leqslant$ 0.55 lead to highly distorted round ends that develop into a cone shape before contact. The contact geometry is that of two sharp cones facing each other, see the focused picture corresponding to $E_{\infty}=0.2$ in Fig. 8. On the contrary, for $E_{\infty}>0.6$ there is an overturning of the protruding drop ends and the contact area is not sharp, and this makes the $\beta$ angle calculation more difficult. At $E_{\infty}=0.7$ the computations show that there is air entrapment as a result of the ends overturning, see Fig. 9. The algorithm fills the gap with the inner fluid, as we have not included the motion of the exterior fluid. This is obviously not physically acceptable and we have not computed scenarios where $E_{\infty}>0.7$. The overturning and trapped air bubble has been previously reported for experimental and numerical studies for a leaky dielectric fluid in a leaky dielectric medium model [14]. This overturning can be attributed to the fact that the drops are about to undergo a jet discharge.

\section{Postcoalescence electrohydrodynamics}

Pair-droplet evolution after coalescence exhibits complex dynamics and a behavioral classification in terms of the $\left(X_{0}, E_{\infty}\right)$ space is considered in this section. In order to have a reference (or catalog) of possible coalesced shapes we first examine the case $X_{0}=0$, i.e., the droplets are tangentially
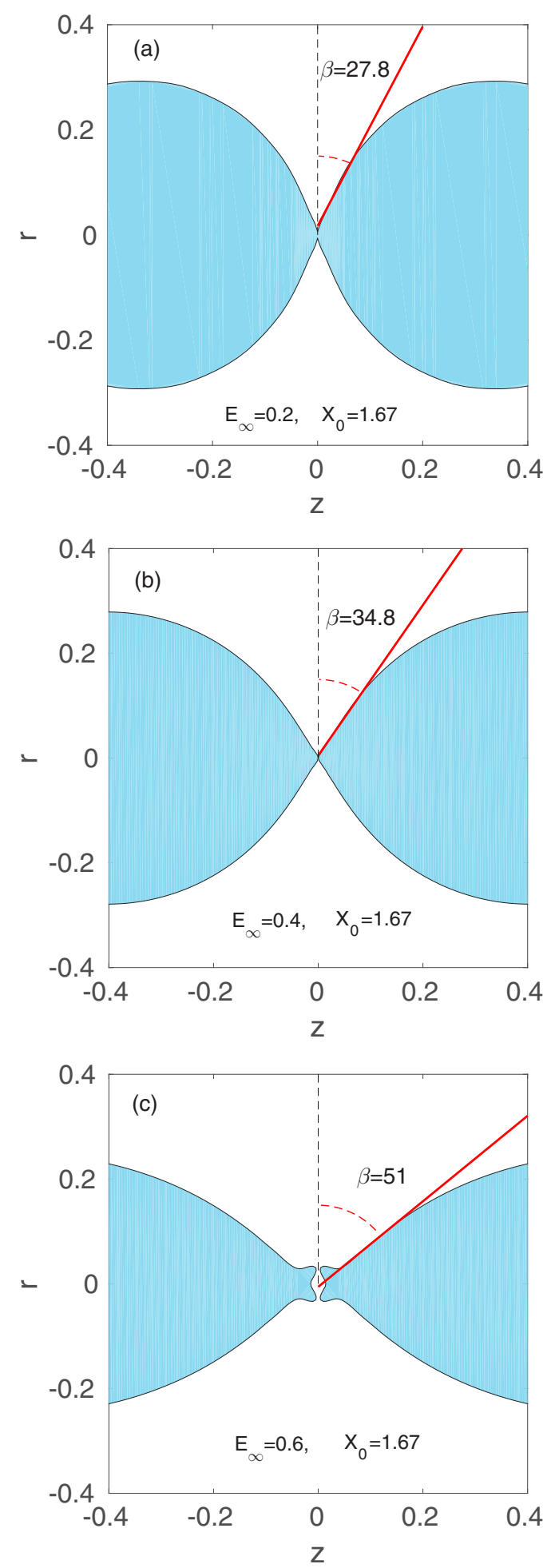

FIG. 8. Calculated cone angles at contact points for $X_{0}=1.67$ and $E_{\infty}=0.2,0.4,0.6$. 

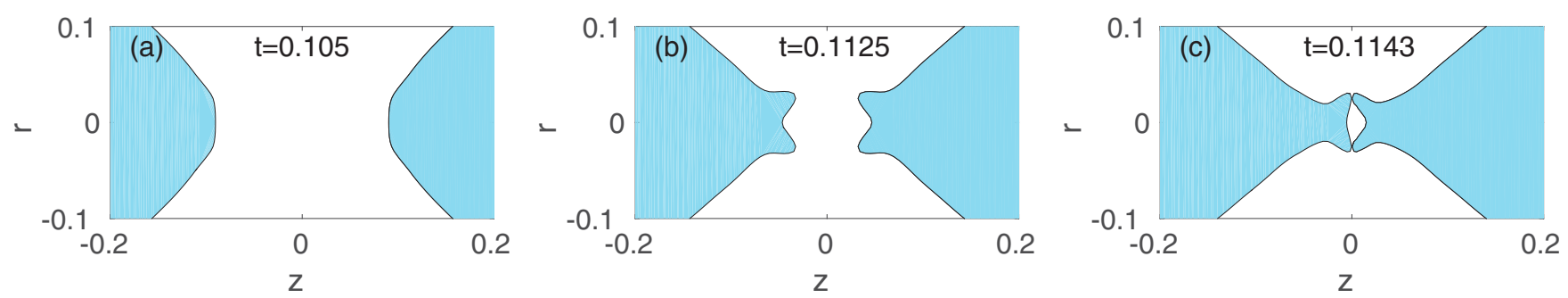

FIG. 9. Zoomed drops facing shapes for $E_{\infty}=0.7$. The individual droplets are about to emit jets and an air bubble is trapped inside the fluid.

touching at $z=0, \beta=0$, and the initial velocity potential is zero. The droplets aspect ratio evolution corresponding to values $E_{\infty} \in[0,0.4]$ is shown in Fig. 10 from which some flow characteristics can be extracted. For times $t<0.05$ all the curves overlap with constant aspect ratio, $a / b=2$, meaning that right after contact all the dynamics occur solely on the bridge region. Within times $0.05<t<0.125$ the curves adopt the same shape with peaks and lows occurring at approximately the same times: The initial disturbance has propagated and reached the droplet end points but the dynamics seem to be almost independent of the electric field intensity. In the time range $t>0.15$ the curve trends open up revealing a strong influence of the electric field intensity on the drop aspect ratio evolution. Table III shows the shapes catalog for various $E_{\infty}$ values at final simulation time, $t_{f}$, and the maximum aspect ratio achieved in the time interval $\left[0, t_{f}\right]$. Inspecting the final drop shape and the maximum aspect ratio for each of the numerical simulations, it is clear that the $X_{0}=0$ case study presents three distinguished regimes: The coalesced droplet oscillates in $E_{\infty} \in[0,0.26]$ and the aspect ratio do not exceed the 2.5 value, $2<a / b<2.5$; in the range $E_{\infty} \in[0.28,0.32]$ the unified droplet elongates enough so that in the recoil process two satellite droplets pinch off and the aspect ratio stays in the interval $2.5<a / b<3$; finally, for $E_{\infty} \geqslant 0.34$ the merged droplet destabilizes and jet emission

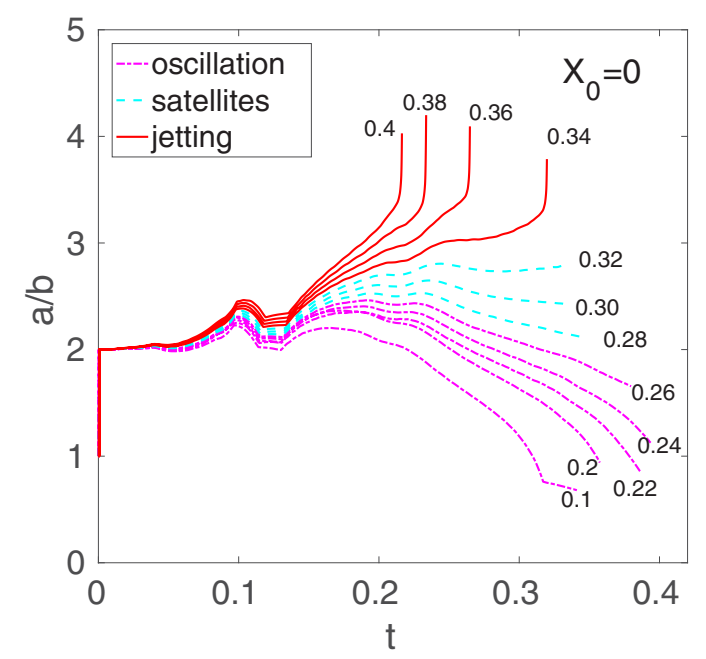

FIG. 10. Aspect ratio $a / b$ evolution after contact for $X_{0}=0$ at indicated $E_{\infty}$ values. The postcoalescence sequence is oscillation, satellites, and jetting. takes place. In what follows, the aspect ratio evolution figures will identify the different regimes using the same color scheme employed at the beginning of this section. Due to short simulated times and zero impact velocities, the numerical results are accurate and stable and thus the $X_{0}=0$ study provides a convenient guide for the regime classification.

Next we discuss the merged droplet aspect ratio behavior corresponding to $X_{0}=0.4, X_{0}=1$, and $X_{0}=1.67$, Fig. 11 . For $X_{0}=0.4$ and $X_{0}=1$, aspect ratios less than $3, a / b \leqslant$ 3 lead to postcoalescence oscillation, whereas for $a / b \approx 3$ satellite pinch-off coalescence mode will occur. If $a / b>3$, then the merged droplet will undergo tip streaming. Regarding $X_{0}=1.67$ the same aspect ratio analysis holds but now the cutting value is $a / b=2.5$. These two cutting values, $a / b=3$ and $a / b=2.5$, are similar to the one corresponding to the $X_{0}=0$ aspect ratio behavior, in terms of the final coalescence mode classification. We can conclude that if coalescence takes place, then the droplet aspect ratio is a good indicator of the postcoalescence drop evolution. Figure 11 shows the the individual droplets aspect ratio evolution when the droplets fail to coalesce and recoil, showing a decrease in this droplet geometric feature.

Finally, we describe several flow characteristics for $X_{0}=$ 1.67. For $E_{\infty} \in[0.2,0.33]$, the drops coalesce forming a thin liquid bridge that develops a tiny protruding ring that has been observed in laboratory experiments. It originates in the collision of the two opposite flows; see, for example, Fig. 12(d). The capillary wave induced by this disturbance propagates through the droplet surface to the end points, leading to two protruding cylinders that eventually stop expanding in the axial direction. Here the competition between surface tension forces and electric stresses determines the final droplet state. If the aspect ratio $a / b$ is below 2.5 , then the central droplet mass will absorb the protruding cylinders; if $a / b$ exceeds 2.5 , then the necks near the central part of the drop shrink and two satellite droplets pinch-off. The break-up time and the size of the daughter droplets is a function of $E_{\infty}$. There is no jet ejection for these range of values. This behavior appears also in the $X_{0}=0$ case for similar $a / b$ values but different $E_{\infty}$ range as can be seen in the shape catalog, Table III. Front profiles for $E_{\infty}=0.29$ before and after coalescence are shown in Fig. 12.

For increased electric field, $E_{\infty} \in[0.34,0.425]$, the postcontact neck grows, but the electric field stress at the boundary acts in a similar way as viscosity. The capillary waves are therefore damped and the drop elongates faster and deforms more, reaching aspect ratios of $a / b \approx 3$. For $E_{\infty}=0.4$ tip 
TABLE III. Shape catalog for $X_{0}=0$. Column $t_{f}$ lists the final simulated time, column $a / b$ lists the maximum aspect ratio achieved during the simulation, and column "Final shape" shows the droplet profile at $t_{f}$.

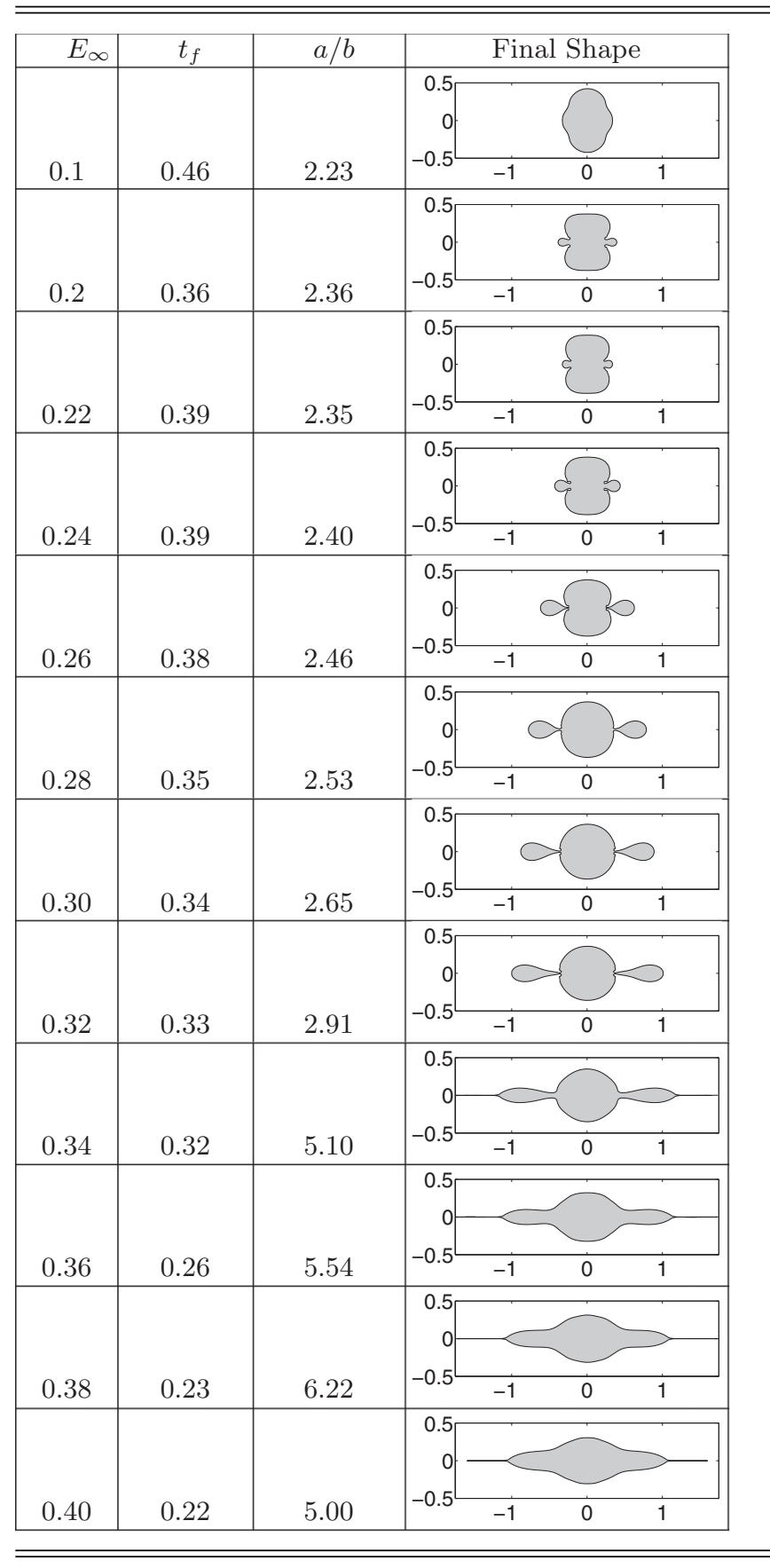

streaming starts at $t=1.37464$ and filaments are ejected at frequencies which range from approximately 0.0005 to 0.002 time units. The volume lost by tip streaming is just $V_{l}=$ 0.000179 , and at the end of the simulation the drop continued in this ejection mode. The aspect ratio of the merged drop before destabilization is $a / b \approx 4$. Drop volume and aspect ratio evolution are shown in Fig. 13, where each peak shown in the onset figure represents one jet ejection. Front profiles corresponding to $E_{\infty}=0.4$ at various times are shown in Fig. 14.
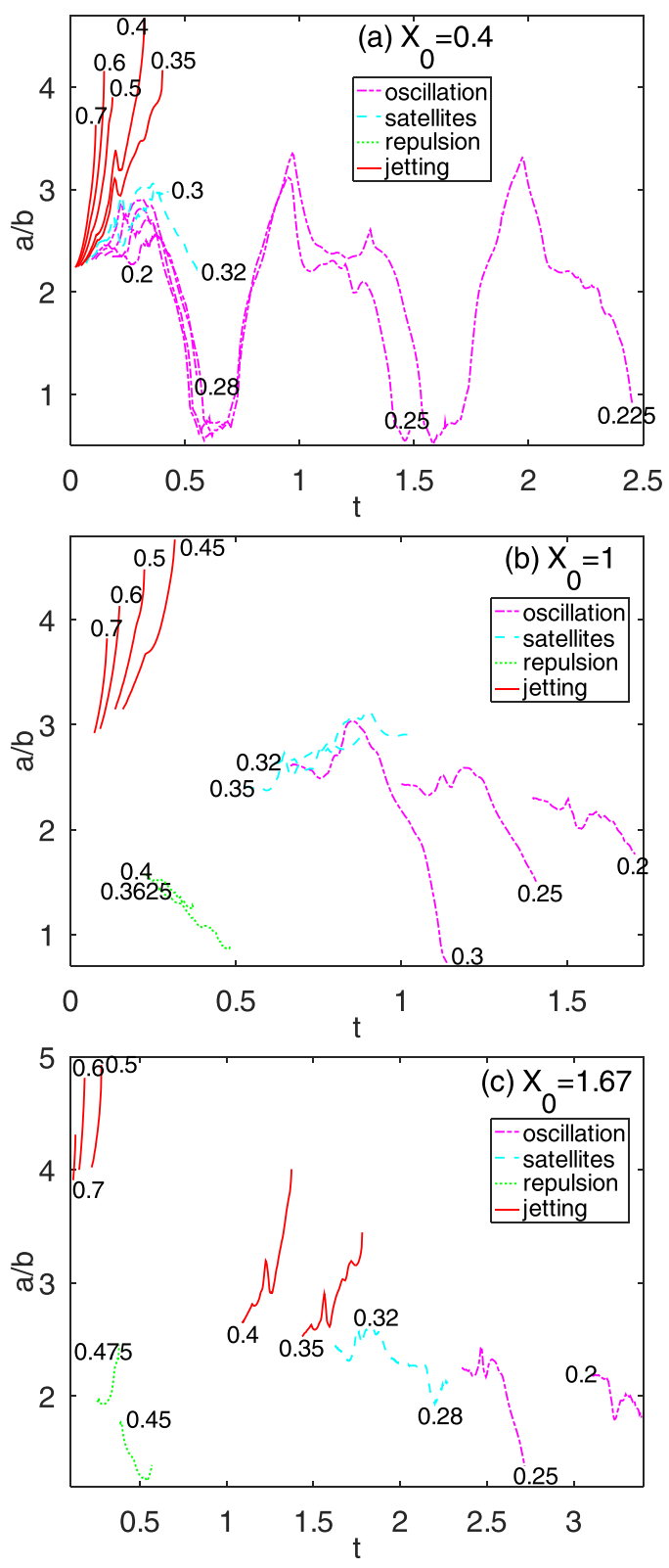

FIG. 11. Evolution of the postcoalesced droplets aspect ratio $a / b$ for various electric fields intensities. Each graph specifies the corresponding $X_{0}$ value.

For $E_{\infty} \in[0.45,0.5)$ the drops fail to coalesce after contact and they recoil. At contact time the unified drop mass has a certain surface charge distribution with zero net charge. When the drop splits in two, each drop carries opposite charges, but they nevertheless repel each other under the effect of the electric direction at the far field. Note that a droplet carrying a positive charge will be moved to the right, whereas it will be translated to the left if it is negatively charged. Individual drops may destabilize and jets are emitted at opposite end points. The evolution of the drop pair is depicted in Fig. 15 at some significant instants for $E_{\infty}=0.45$.

With $E_{\infty} \in[0.5,0.7]$ and before merging the droplets are about to burst. After they make contact the neck region has no time to expand and the unified droplet deforms very fast, reaching aspect ratios of $a / b>4$ before a thin filament is 

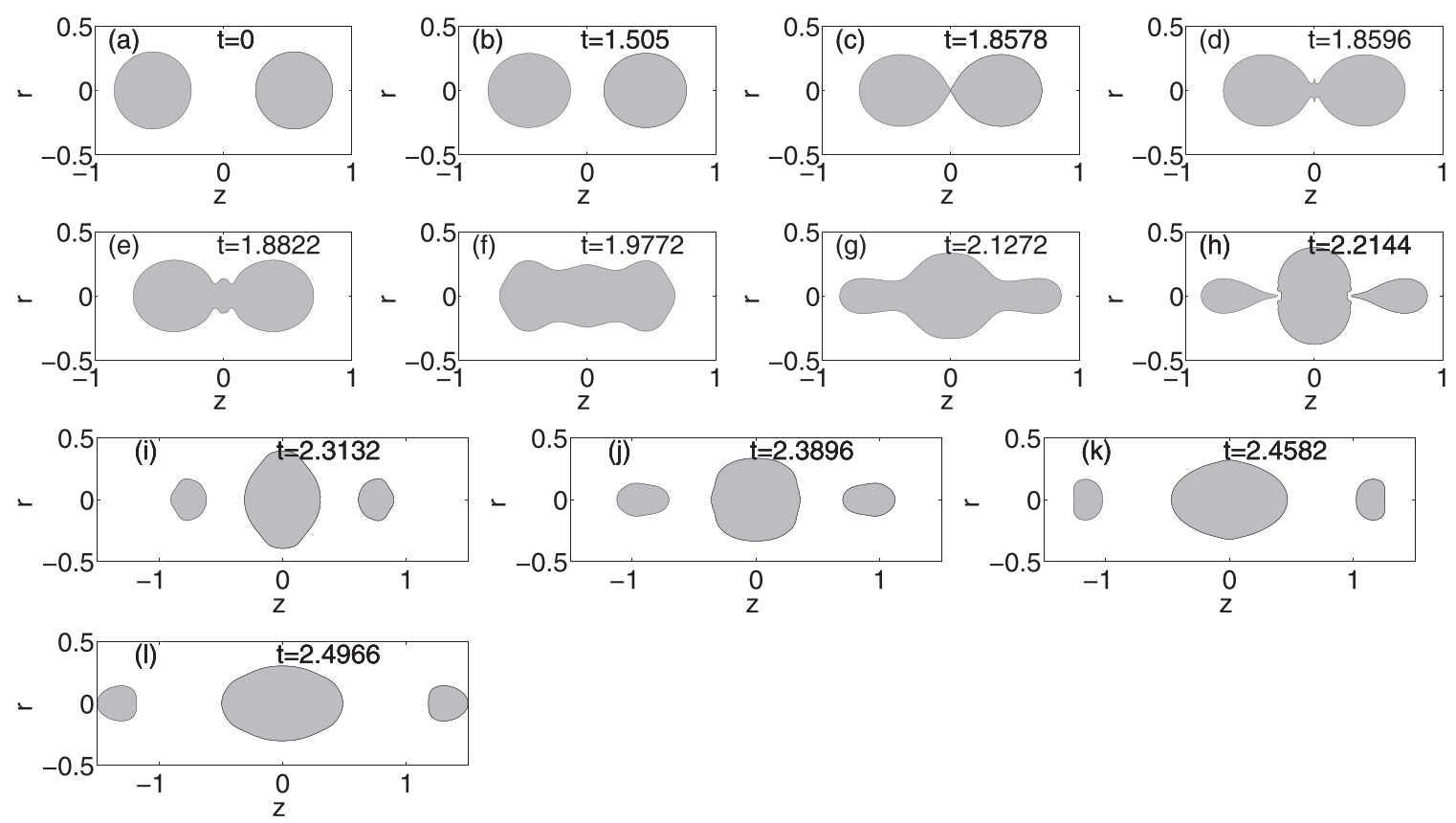

FIG. 12. Front profiles at various times for $X_{0}=1.67$ and $E_{\infty}=0.29$. At $t=2.2144$ two opposite charged satellite droplets pinch-off.

ejected. These high values of the nondimensionless electric field strength may not correspond to feasible physical values.

The postcoalescence modes and underlying physics that have been observed for $X_{0}=1.67$ can be summarized as follows:

(1) No coalescence within $T=4$ for $E_{\infty}<0.2$.

(2) With $E_{\infty} \in[0.2,0.27]$ droplets oscillate and then merge into a bigger droplet at a low average velocity $V_{M} \approx$ 0.1 . The values of the electric field intensity are moderate and the droplet deformation in the axial direction is restored early on by surface tension. The alternating play of forces makes the droplet oscillate and change from horizontal capsule shapes to vertical ones.

(3) For $E_{\infty} \in[0.28,0.33]$ the droplets come together oscillating with the impact average velocity $V_{M} \approx 0.2$, causing a visible droplet flow upward. The generated wave will propagate through the merged drop surface, not being sufficiently

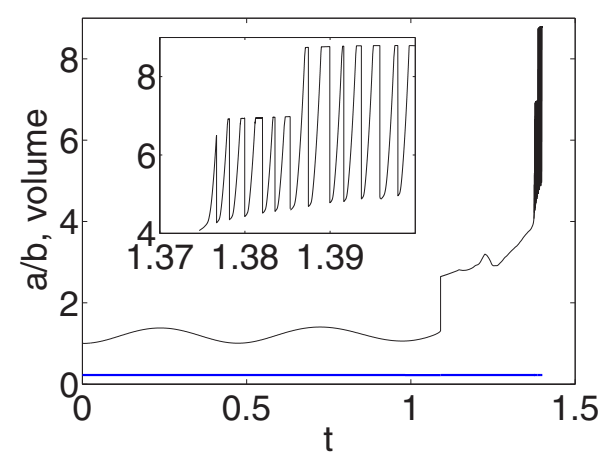

FIG. 13. Aspect ratio evolution $a / b$ for $E_{\infty}=0.4$ before and after coalescence time (black) and drop volume evolution (blue). Jet ejection frequencies can be seen in the inset figure. At $t=1.0409$ coalescence takes place and conservation of volume holds after passing through the singularity. damped by the still moderate electrical stresses, while the electrical stress expands the droplet in the axial direction. As a consequence of these two effects, two protruding cylinders appear at the droplet ends. Surface tension then acts to restore shapes that minimize surface energy, causing the necks at the base of the cylinders to collapse. Two equal size daughter droplets pinch-off.

(4) When $E_{\infty} \in[0.34,0.425]$ drops oscillate and coalesce into a bigger droplet at $V_{M} \approx 0.25$. The electric field intensity is now big enough to damp capillary waves and to produce droplet deformations up to aspect ratios $a / b \approx 3$. Protruding cylinders rapidly adopt Taylor cone angles at their ends from which intermittent tip streaming occurs.

(5) With $E_{\infty} \in[0.45,0.50)$ droplets deform significantly as they approach each other, and the impact average velocity rises to $V_{M} \approx 0.5$. The geometry of the contact region and other combined processes are such that after a short coalescence the drops move apart. This mechanism will be explained in more detail in the next subsection.

(6) For $E_{\infty} \geqslant 0.5$ electrical stresses dominate over surface tension forces making the droplets deform significantly as they approach each other. Droplets are nearly about to burst prior to make contact and the created bridge will not expand. Very soon after contact a permanent filament is ejected from the end points.

Similar analysis has been carried out for other $X_{0}$ values. For increasing values of $E_{\infty}$ the same postcoalescence sequence has been obtained: oscillation, satellites, and jetting. The repulsion mode shows up whenever the transition regime occurs. More comments on this will be given in the next subsection.

\section{Repulsion versus coalescence mechanism}

The dynamics of the system are controlled by the competition of inertial forces, surface tension forces, and electric 

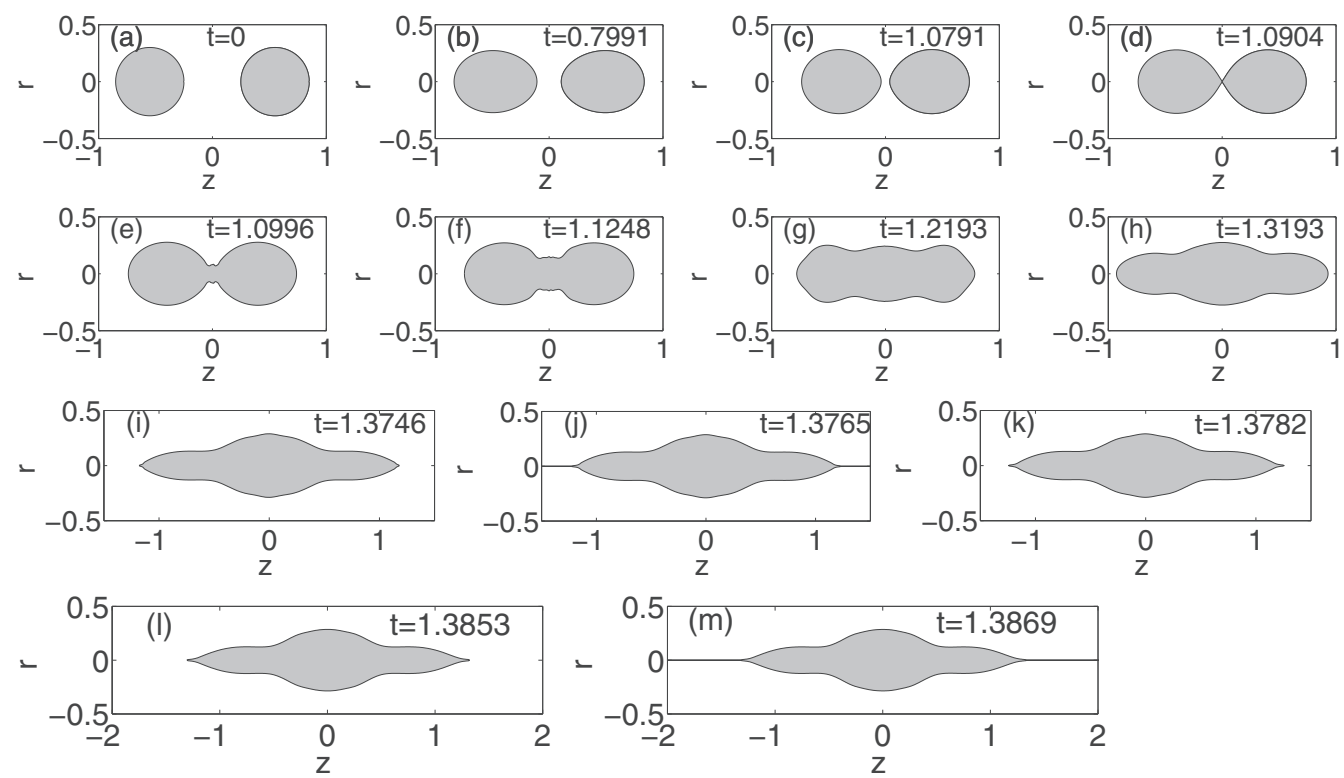

FIG. 14. Front profiles at various times for $X_{0}=1.67$ and $E_{\infty}=0.4$. At $t=1.3746$ the protruding cylinders develop Taylor cones from which tip streaming occurs.

stresses, all acting in the normal direction to the free surface. Under the action of a uniform electric field an isolated and neutral droplet will become a prolate spheroid, positively charged on its right face and negatively charged on its left face. The individual droplet will act as a dipole source [1]. On a droplet pair system the dipole-dipole forces are superposed to the far field electric forces, creating more intricate motions. As we have shown, even prior to coalescence, two distinct flows are present. In one regime deforming and restoring forces sequentially switch, whereas in the other regime electrical forces are clearly dominant. It is in the transition between these patterns where droplet repulsion takes place due to a combination of factors. The electric field intensity range within the transition zone and the associated cone angles are different for each $X_{0}$, which indicate that recoiling is not only caused by a pure geometric and static feature as the contact cone angle $\beta$.
Next we analyze in more detail force interactions for the studied $X_{0}$ values.

(i) For $X_{0}=0.4$ and all $E_{\infty}$ values the droplet distance is small enough such that the dipole-dipole forces are dominant and the droplets just translate and deform approaching each other without oscillating. The droplet aspect ratio do not go over 1.2 and neither $z_{A}$ or $z_{C}$ oscillates but move with increasing velocity that seems to blow up when $z_{A} \rightarrow 0$; we can also observe that $z_{A}$ moves faster that $z_{C}$. All these observations show that there is only one regime prior to coalescence (electric forces dominates over surface tension restoring forces for all $E_{\infty}$ ). This fact can also be seen on the curve $t_{c}$ versus $E_{\infty}$, where only one scaling law is present. For this droplet separation no repulsion is detected.

(ii) For $X_{0}=1$ and $E_{\infty} \in[0.2,0.35]$ dipole-dipole forces are weak initially and droplets oscillate showing the regime where restoring forces compete against electrical deforming
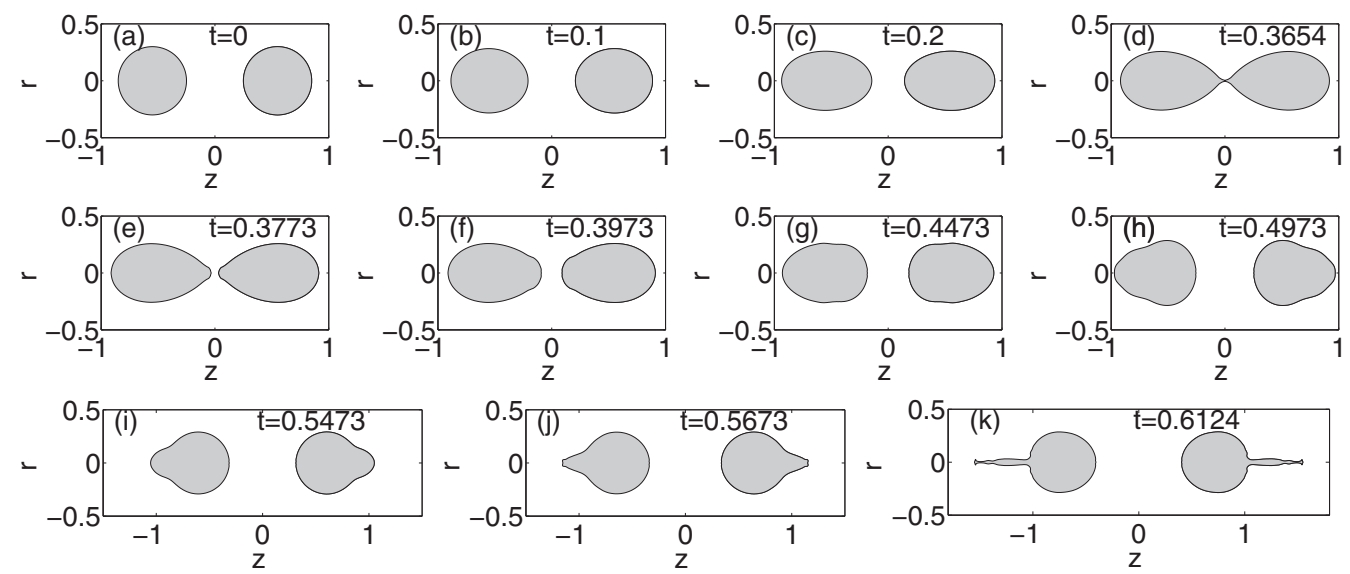

FIG. 15. Front profiles at various times for $X_{0}=1.67$ and $E_{\infty}=0.45$. After first contact at $t=0.3654$ the droplets recoil and fail to coalesce. The droplets carry opposite charges and at $t=0.5673$ jet emission takes place. 

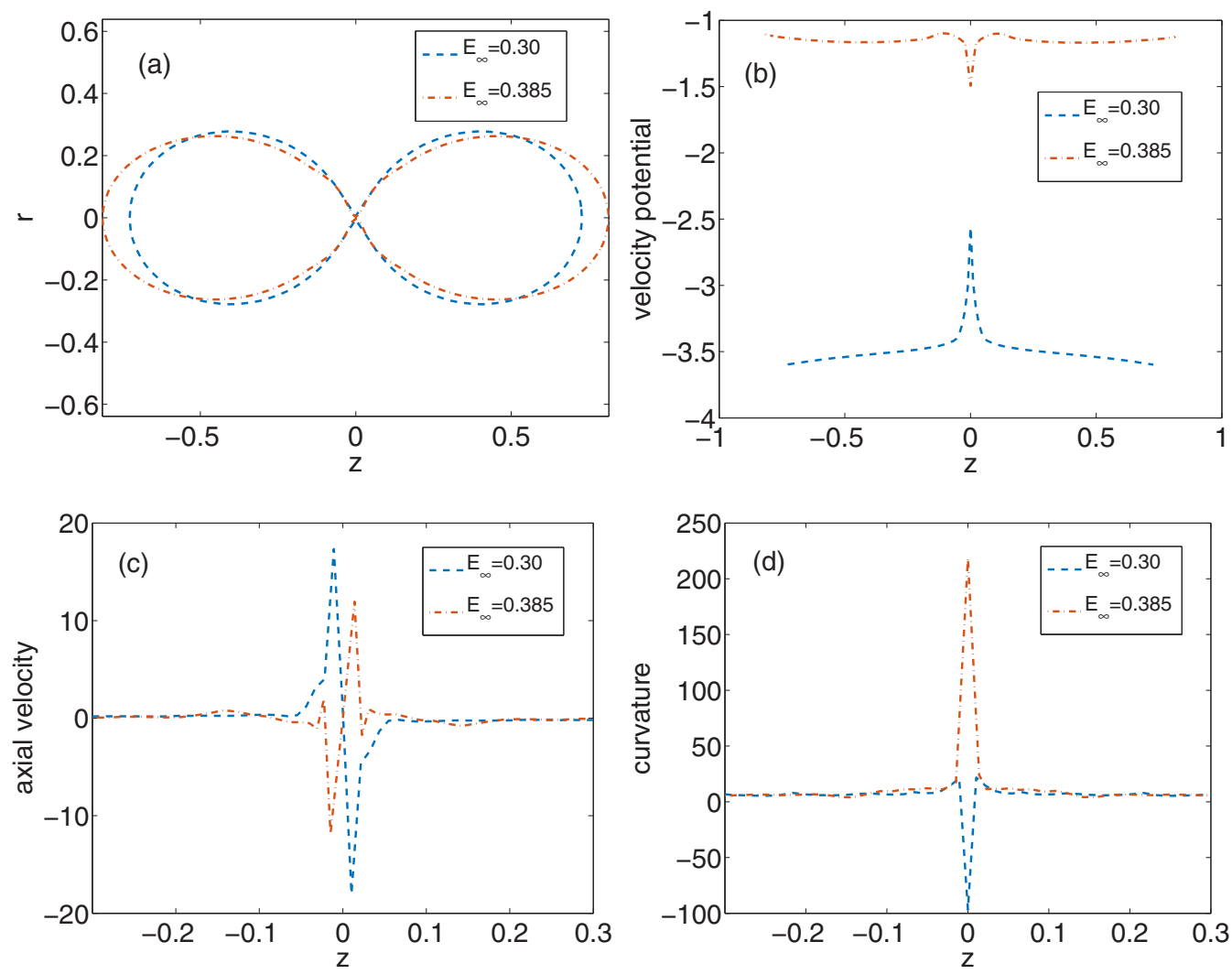

FIG. 16. Comparison between flow variables for repulsion, $E_{\infty}=0.385$, versus coalescence, $E_{\infty}=0.3$ at contact time. The rescaled separation is $X_{0}=1$. Panel (a) shows that the repulsion front profile is more deformed than the coalescence profile and panel (b) shows velocity potentials. In panel (c) the opposite axial velocity behavior is depicted and panel (d) shows the opposite curvature signs at contact point.

forces. When $z_{A} \leqslant 0.08$ dipole forces start acting and electrical deformation is enhanced, reaching aspect ratios of $a / b=$ 1.32 for $E_{\infty}=0.35$. For $E_{\infty} \in[0.5,0.7]$ electrical forces dominates, $z_{A}$ and $z_{C}$ move very fast toward coalescence, mainly because they are about to undergo tip streaming.

The transition zone, $E_{\infty} \in[0.36,0.40]$, corresponds to the repulsion regime. The numerical results show that combined processes act simultaneously: (1) The local geometry near the contact point is such that the neck curvature becomes highly positive and the flow reverses direction. This is also possible because the average impact velocity is still moderate. (2) At the droplet ends the axial velocity is positive on the right end and negative on the left end, which enhances the recoiling process. (3) Once the droplets fall apart the deformed drop shape in the axial direction triggers surface tension forces to restore spherical shapes. Moreover, the calculated charges are equal with opposite signs, but in this range of $E_{\infty}$ values the electrical field overcomes the droplet attraction force pushing the droplets further apart.

To support the above statements we show in Fig. 16 the flow variables for $E_{\infty}=0.30$ and $E_{\infty}=0.385$, which correspond to coalescence and recoil, respectively. The distinct behavior of front shapes, velocity potentials, axial velocities, and front curvature is clear. Front curvatures at the neck have opposite signs in both curves, see Fig. 16(d), which in turn makes the axial velocities direct the flow in opposite directions, see Fig. 16(c). Note also that away from the neck, at the droplet end regions, the axial velocities also play a roll in the coalescence or recoiling regime: They also exhibit opposite signs.

(iii) In the case $X_{0}=1.67$ the same previous arguments apply, and here the first regime corresponds to $E_{\infty} \in[0.2,0.4]$ as the drops come together oscillating, whereas in $E_{\infty} \in$ $[0.5,0.70]$ electric deformation prevails. In the transition zone $E_{\infty} \in[0.45,0.5)$ repulsion occurs. The fact that the repulsion zone lays between red triangles is not an issue, as red triangles just indicate that the merged droplet will finally undergo jet emission, which is justified by aspect ratios getting bigger than 3 . We can compare the dynamics of the point in the parameter space $(1,0.35)$ and $(1.67,0.35)$ and observe the

TABLE IV. Repulsion mode convergence analysis with respect the time step for $X_{0}=1$. The electric field intensity, $E_{\infty}$, the time step, $\Delta t$, the coalescence time, $t_{c}$, and the postcoalescence mode are listed.

\begin{tabular}{llcc}
\hline \hline$E_{\infty}$ & \multicolumn{1}{c}{$\Delta t$} & $t_{c}$ & Mode \\
\hline 0.375 & 0.001 & 0.2220 & Repulsion \\
0.375 & 0.0001 & 0.2217 & Repulsion \\
0.385 & 0.001 & 0.2049 & Repulsion \\
0.385 & 0.0001 & 0.2044 & Repulsion \\
0.40 & 0.001 & 0.1853 & Repulsion \\
0.40 & 0.0001 & 0.1849 & Repulsion \\
\hline \hline
\end{tabular}


different aspect ratios after coalescence which is in accordance with the fact that the first point is in the satellite mode and the second one in the jetting mode. We noticed that, for this $X_{0}$ value, the jetting behaviors in $E_{\infty} \in[0.35,0.4]$ and $E_{\infty} \in[0.5,0.7]$ are very different but we refused to assign a different color and symbol as for practical purposes tip streaming will occur. Apart from the droplet shapes, the main difference is that tiny droplets are sequentially ejected from the Taylor cones in $E_{\infty} \in[0.35,0.4]$. Within $E_{\infty} \in[0.5,0.7]$ the droplets are about to destabilize prior to merging and the unified mass will soon after emit a long jet. Recall that for isolated droplets of radios $R=0.3$ the critical electrical field intensity for droplet destabilization is $E_{\infty}=0.48$.

Although convergence of the algorithm has been sufficiently established in previous works, we want to show that the repulsion mode is not an artifact of the numerical approximation. To this aim, and because the time step controls how the algorithm passes through singular events (see Ref. [12]), we have run simulations for $X_{0}=1$ taking $\Delta t=0.001$ and $\Delta t=0.0001$. The results are shown in Table IV where we list the value of $E_{\infty}$, the time step $\Delta t$, the coalescence time $t_{c}$, and the resulting coalescence mode. We can conclude that the repulsion mode is stable.

\section{E. Comparison with laboratory experiments}

Experimental studies reported in Ref. [21] and Ref. [20] will be used to compare numerical and experimental results. In Ref. [21] pairs of Milli-Q water droplets immersed in a hexadecane oil emulsion are pumped into a microchannel and subjected to an ac electrical current. In their work they examine the stability diagram for stable, coalescing, and partial merging (repulsion) with respect the rescaled droplet separation and nondimensional electric field. Stable droplet pairs refers to lack of merging within a certain elapsed time. Despite the various differences between the experimental setting and the model assumptions, we want to examine the extent to which the numerical results agree with the experimental findings. The main discrepancies should be attributed to the fact that the model does not include the effects of the surrounding oil flow: The oil will slow the dynamics and mitigate the propagation of capillary waves. Figure 2 in Ref. [21] shows photographs of droplet pairs evolution for rescaled distances $X_{0}=1.12, X_{0}=0.80$, and $X_{0}=0.54$ when an ac electric field of $E=2739 \mathrm{kV} / \mathrm{m}$ at a frequency of $10 \mathrm{kHz}$ is applied. They found that for $X_{0}=0.80$ the repulsion mode occurs. To carry out the numerical simulation, the corresponding nondimensional value has to be calculated. Taken reported values, $R_{0}=20 \mu \mathrm{m}$ and $\gamma=0.0055 \mathrm{~kg} / \mathrm{m}^{3}$, the characteristic scale is $E_{0}=\left(\frac{2 \gamma}{\epsilon R_{0}}\right)^{1 / 2}=7538 \mathrm{kV} / \mathrm{m}$, and $E_{\infty}=0.36$.

A new set of simulations have been performed for $X_{0}=0.8$ and $E_{\infty} \in[0.25,0.40]$, taking increments of 0.01 units. The coalescence time $t_{c}$ versus $E_{\infty}$ is plotted in Fig. 17, where it can be observed that the curve jumps at around $E_{\infty}=$ 0.30 . Indeed, for $E_{\infty}=0.31$ the droplets fail to coalesce and repel each other; see the droplet profiles at selected times in Table V. This result supports the hypothesis that the repulsion mechanism occurs in the transition regime and that a method to predict droplet repulsion has been provided. If we consider

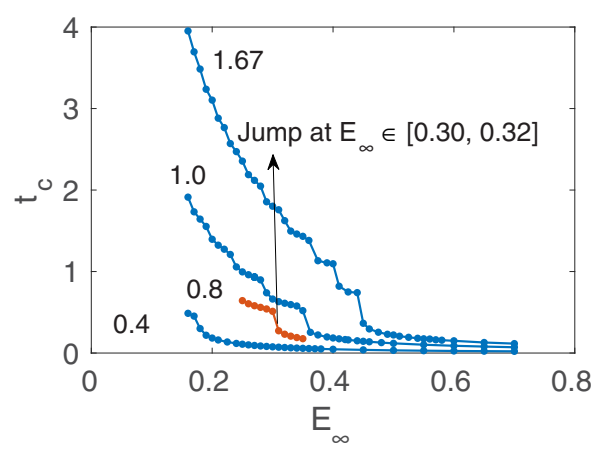

FIG. 17. Coalescence times $t_{c}$ versus $E_{\infty}$ for $X_{0}=0.8$. The curves corresponding to $X_{0}=0.4,1,1,67$ has been included as a reference. The transition zone between regimes occurs in the interval $E_{\infty} \in[0.3,0.32]$ where recoiling takes place.

that the characteristic timescale for the experiment is $1 \mathrm{~ms}$, then the times until the droplets first touch and repel each other are in good agreement. Due to the lack of an exterior fluid in the mathematical model, the droplets post repulsion fall apart, as there is no damping of the surface oscillations.

An attempt to compare numerical results with the experimental work reported in Ref. [20] has also been made. Their experimental setting consists in two needles separated a distance $d$, from which two water droplets are injected in air. Our $X_{0}$ parameter cannot be derived from their $d$, as droplets are brought together by a voltage difference and the injection speed. They mainly focus on and discuss the critical cone angle $\beta$ that separates coalescence from the recoiling regime. They state that "the cone angle $\beta$ is positively correlated

TABLE V. Three-dimensional renderings of the computed droplets profiles for $X_{0}=0.8$. At $t=0.27$ the droplets touch and immediately recoil. For this value of the electric field, $E_{\infty}=0.31$, the individual droplets are stable.

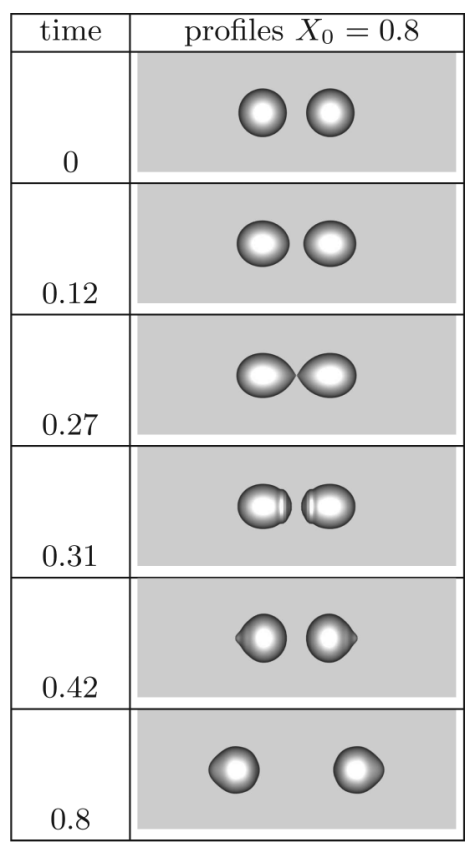


with the applied voltage and negatively correlated with the needle separation." The numerical results also show that the cone angle increases with $E_{\infty}$ but increases as well with $X_{0}$ at least for $E_{\infty}>0.45$. For $E_{\infty}<0.45$ the sign of the correlation is not clear, see Fig. 6. In their Fig. 4 the value of the electrocapillary number that separates coalescence from recoiling is $\sqrt{\mathcal{E}_{c}}=0.7$. Note that $\sqrt{\mathcal{E}_{c}}=\sqrt{2} E_{\infty}$, which leads to $E_{\infty}=0.5$. For $X_{0}=1.67$ we found recoiling in the range $E_{\infty} \in[0.45,0.5]$ and the cone angle $\beta \in[38.5,47.5]$, which qualitatively agrees with their experimental results. Here the main difference between experimental and numerical settings is that the laboratory water droplets are constantly attached to the needles, while in the numerical simulations the droplets are freely suspended in air. This clearly makes a direct comparison difficult.

\section{MULTIDROPLET ELECTROHYDRODYNAMIC}

In this section we present numerical results for the electrohydrodynamic interaction of multiple droplets. Here the possible number of geometric configurations is huge and thus we focus attention on the laboratory experiments reported in Ref. [21]. In these experiments the droplets are immersed in an oil phase and confined in a microchannel, limiting the droplet movement in the radial direction. Nevertheless, the axial symmetry holds and these experiments can be used as a reference.

Two case studies have been investigated:

(a) Six droplets of radii $R_{0}=0.3$ uniformly placed at an initial distance $D_{0}=0.5$.

(b) Nine droplets of radii $R_{0}=0.3$ in a nonuniform array.

It is important to note that in the numerical experiments the droplets are all present at $t=0$, whereas in the laboratory experiments the droplets are fed into the microchannel at times $t>0$. This difference has to be taken into account when comparing the results.

\section{A. Six-droplet train}

Six droplets of radii $R_{0}=0.3$ were placed at a distance of $D_{0}=0.5$, and thus $X_{0}=1.67$ for any of the droplet pairs. The applied electric field was chosen as $E_{\infty}=0.4$. The computational fixed domain is now $\Omega_{D}=[-3.5,3.5] \times$ $[-1,1]$, mesh size is $\Delta r=\Delta z=0.005$, and the time step ranges from $\Delta t=0.001$ initially to $\Delta t=10^{-5}$. The initial node spacing on the free fronts is $d s=0.02$. To study how the presence of several drops enhance the electric field action, Fig. 18 displays the aspect ratio, $a / b$, evolution for each of the six droplets. The six droplets are numbered from left to right, and we observe that the left and right most droplets (drops 1 and 6) oscillate with a maximum deformation of $a / b=1.4$. This agrees with the recorded deformation for the isolated droplet pair $\left(X_{0}, E_{\infty}\right)=(1.67,0.40)$. The most inner droplets, drops 3 and 4 , oscillate with a larger deformation, reaching values of $a / b=1.6$. Symmetry with respect $z=0$ is also very well preserved. All of these results are in good agreement with theoretical analyses [17] that predict the magnification of the electrical field due to dipole action.

The evolution of the droplet train is shown in Fig. 19. We have chosen the three-dimensional version of the computed profiles to have a better sense of reality. Although the

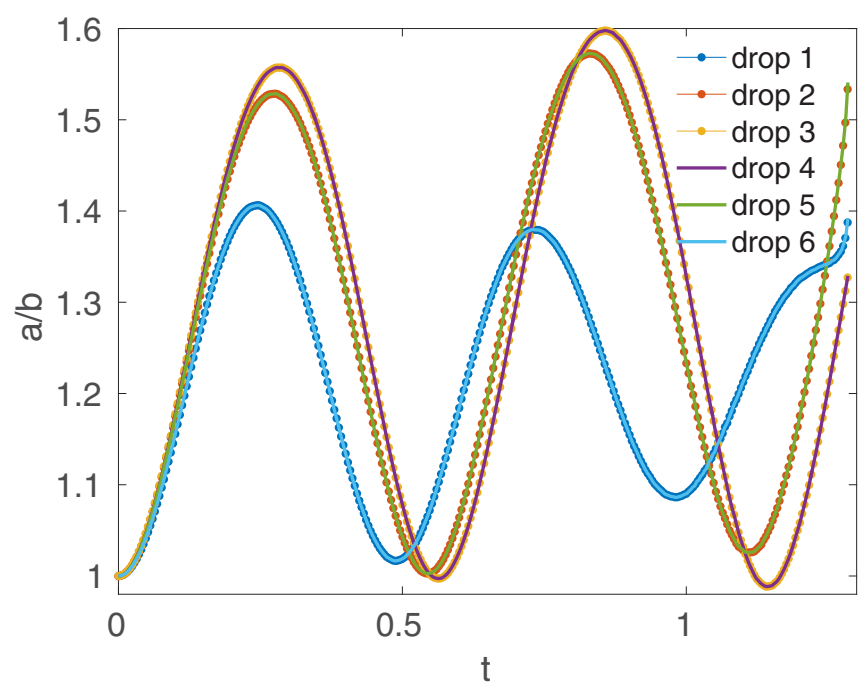

FIG. 18. Six droplets aspect ratio $a / b$ evolution for $E_{\infty}=0.4$ before first coalescence. Droplets 1 and 6 undergo same deformation. Same behavior for droplets 2 and 5 and droplets 3 and 4 .

maximum droplet deformation is recorded in droplets 3 and 4 , coalescence is instead initiated at the outer droplets at time $t=1.2857$ and continued at $t=1.3648$; see Figs. 19(d) and 19(f), respectively. This is in surprisingly good agreement with the propagation of the coalescence front, from right to left, reported in experiments [21] [their Fig. 5(b)]. The simulation is finished at $t=1.4$, where the merged front is destabilized and ready to initiate tip streaming.

\section{B. Nine-droplet train}

The nine-droplet train geometrical configuration chosen here was modeled after the experiments reported in Ref. [21] and in particular their Fig. 5(a). Moreover, we wanted to study the evolution of a droplet train that was not uniformly spaced initially. The radii of the nine droplets is $R_{0}=0.3$ and the initial distances are $D_{1,2}=D_{7,8}=0.5, D_{2,3}=D_{3,4}=$ $D_{8,9}=0.1, D_{4,5}=0.3$, and $D_{5,6}=D_{6,7}=0.2$, where $D_{i, j}$ is the initial distance from drop $i$ to drop $j$. The discretization parameters used here are the same as above, except that the fixed domain is taken as $\Omega_{D}=[-5,3.75] \times[-1,1]$ and the initial time step is $\Delta t=10^{-4}$. The smaller time step was a consequence of the expected faster coalescence.

In Fig. 20 the evolution of each droplet aspect ratio is shown only until the first coalescence of droplets 2,3 , and 4 at time $t=0.0281$, as the cascade of merging events complicates the droplet numbering. The only droplet that can be followed throughout is number 1 , which stays isolated until the end of the simulation. Inspecting Fig. 20, we see that the drops that undergo maximum deformations are number 3 , followed by 4 and 2 . The minimum deformation corresponds to droplet 1 initially but it reaches a value of $a / b \approx 1.2$ before being absorbed by the main front at $t=0.1213$. None of the nine droplets exhibit oscillations before merging due to the initial short distances between most of the droplets and the fast coalescence events. 


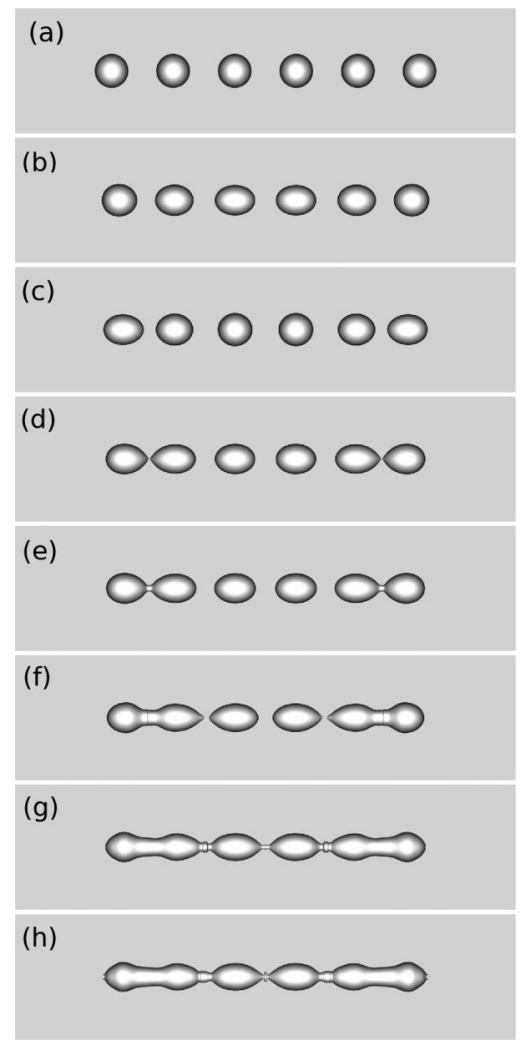

FIG. 19. Three-dimensional renderings of six droplets front profiles for $E_{\infty}=0.4$ at the following times: (a) $t=0$, (b) $t=1$, (c) $t=$ 1.2 , (d) $t=1.2858$, (e) $t=1.3001$, (f) $t=1.3648$, (g) $t=1.3985$, and (h) $t=1.4099$. The horizontal axis is $z \in[-4,4]$ and the vertical axis is $r \in[-0.5,0.5]$. Note that the coalescence front propagates inward.

Finally, Fig. 21 depict the train evolution and front profiles at several times of interest. Here the coalescence cascade is initiated at $t=0.0281$ by the three droplets separated by the shorter initial distance 0.1 , followed by droplets 8 and 9 , Figs. 21(b) and 21(c), respectively. The next drops to join are

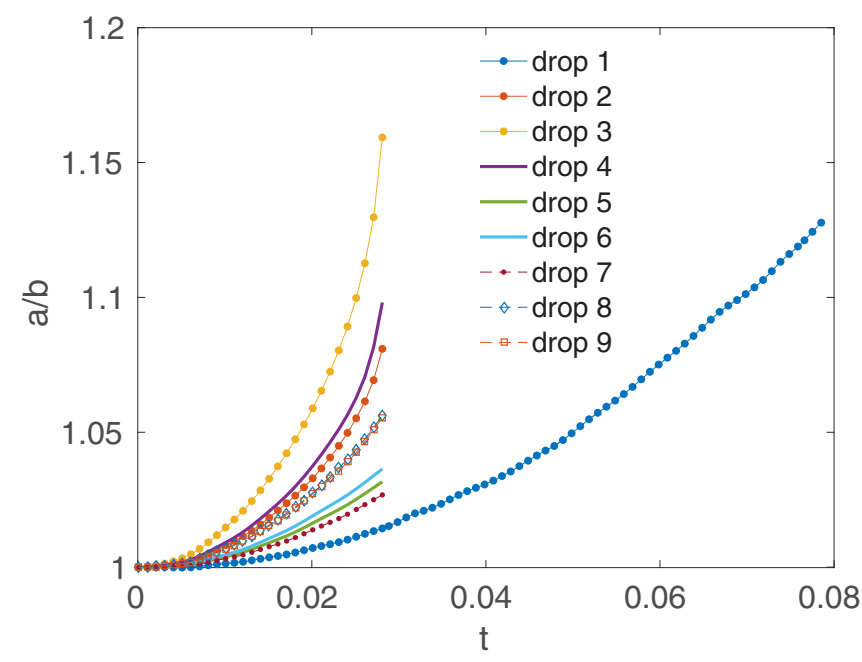

FIG. 20. Nine droplets aspect ratio $a / b$ evolution for $E_{\infty}=0.4$ before first coalescence. All droplets deform differently.

\section{(a)

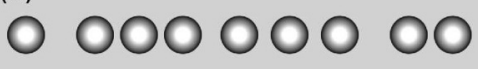

(b)

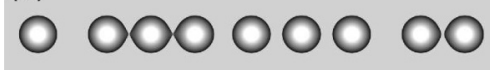

(c)
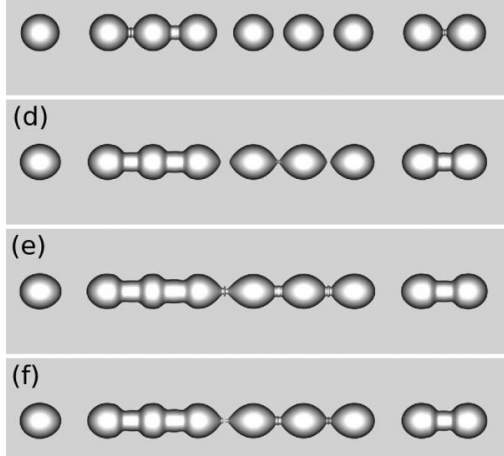

(g)

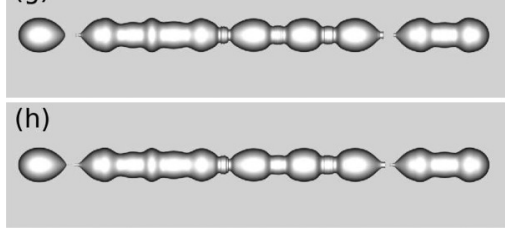

FIG. 21. Three-dimensional renderings of nine droplets front profiles for $E_{\infty}=0.4$ at the following times: (a) $t=0$, (b) $t=$ 0.0281 , (c) $t=0.0488$, (d) $t=0.0785$, (e) $t=0.0850$, (f) $t=$ 0.0910 , (g) $t=0.1176$, and (h) $t=0.1213$. The horizontal axis is $z \in[-5,4]$ and the vertical axis is $r \in[-0.5,0.5]$. Coalescence fronts start propagating where the droplets are closer.

number 5 and 6 [Fig. 21(d)]. The coalescence front propagates until $t=0.1176$ where the central fluid mass is about to emit jets [Fig. 21(g)] that are responsible for the final droplet train connection.

\section{CONCLUSIONS}

Electrohydrodynamic free boundary problems with axial symmetry have been investigated using a coupled boundary integral-level set algorithm. Singular flow events, namely fluid break-up and coalescence, are easily handled, the computations successfully capturing these topological changes. For a pair of droplets, the simulations reveal four distinct coalescence modes depending on the $\left(X_{0}, E_{\infty}\right)$ parameter space. In particular, this simple model reproduces the experimentally observed droplet repulsion seen after a short contact time. A method for predicting the parameter values for which repulsion occurs has been proposed. The electrohydrodynamics of a train of water droplets has also been analyzed and the numerical results compared with laboratory experiments in microchannels. Even though the mathematical model does not include the exterior fluid flow, the numerical results qualitatively predict multiple droplet behavior. 


\section{ACKNOWLEDGMENTS}

This work was supported in part by the Applied Mathematics Program of the U.S. DOE Office of Advanced Scientific Computing Research under Contract
No. DE-AC02-05CH11231. The second author was also supported by the Spanish Project MTM2017-87162-P. The first author was funded by Spanish Project MTM2017-87697-P. The three-dimensional renderings were made by F. Villalon with Blender.
[1] G. I. Taylor, Disintegration of water drops in an electric field, Proc. R. Soc. Lond. Ser. A 280, 383 (1964).

[2] R. L. Grimm and J. L. Beauchamp, Dynamics of field induced droplet ionization: Time-resolved studies of distortion, jetting and progeny formation from charged and neutral methanol droplets exposed to strong electric fields, J. Phys. Chem. B 109, 8244 (2005).

[3] E. Giglio et al., Shape deformation of surface-charged microdroplets, Phys. Rev. E 77, 036319 (2008).

[4] O. A. Basaran, T. W. Patzek, R. E. Benner, and L. E. Scriven, Nonlinear oscillations and breakup of conducting, inviscid drops in an externally applied electric field, Ind. Eng. Chem. Res. 34, 3454 (1995).

[5] R. T. Collins, J. J. Jones, M. T. Harris, and O. A. Basaran, Electrohydrodynamic tip streaming and emission of charged drops from liquid cones, Nat. Phys. 4, 149 (2008).

[6] J. C. Burton and P. Taborek, Simulations of Coulombic Fission of Charged Inviscid Drops, Phys. Rev. Lett. 106, 144501 (2011).

[7] M. Garzon, L. J. Gray, and J. A. Sethian, Numerical simulations of electrostatically driven jets from non-viscous droplets, Phys. Rev. E 89, 033011 (2014).

[8] S. T. Thoroddsen, K. Takehara, and T. G. Etoh, On the coalescence speed of a pendant and sessile drop, J. Fluid Mech. 527, 85 (2005).

[9] F. H. Zhang, E. Q. Li, and S. T. Thoroddsen, Satellite Formation During Coalescence of Unequal Size Drops, Phys. Rev. Lett. 102, 104502 (2009).

[10] A. Menchaca-Rocha, A. Martinez-Davalos, R. Nunez, S. Popinet, and S. Zaleski, Coalescence of liquid drops by surface tension, Phys. Rev. E 63, 046309 (2001).

[11] F. Blanchette and T. P. Bigioni, Partial coalescence of drops at liquid interfaces, Nat. Phys. 2, 254 (2006)

[12] M. Garzon, L. J. Gray, and J. A. Sethian, Electrodynamic coalescence of droplets using an embedded potential flow model, Phys. Rev. E 97, 033112 (2018).

[13] J. C. Baygents, N. J. Rivette, and H. A Stone, Electrodynamic deformation and interaction of drop pairs, J. Fluid Mech. 368 , 359 (1998).

[14] S. Mhatre, S. Deshmukh, and R. M. Thaokar, Electrocoalescence of a drop pair, Phys. Fluids 27, 092106 (2015).

[15] X. Chen, Y. Song, D. Li, and G. Hu, Deformation and interaction of droplet pairs in a microchannel under ac electric fields, Phys. Rev. Appl. 4, 024005 (2015).

[16] K. Adamiak, Interaction of two dielectric or conducting droplets aligned in the uniform electric field, J. Electrost. 51-52, 578 (2001).
[17] W. H. Davis, Two charged spherical conductors in an uniform electric field: Forces and field strength, Q. J. Mech. Appl. Math. 17, 499 (1964).

[18] P. R. Brazier-Smith, Stability and shape of isolated and pairs of water drops in an electric field, Phys. Fluids 14, 1 (1971).

[19] W. D. Ristenpart et al., Non-coalescence of oppositely charged drops, Nat. Lett. 461, 377 (2009).

[20] J. C. Bird, W. D. Ristenpart, A. Belmonte, and H. A. Stone, Critical Angle for Electrically Driven Coalescence of Two Conical Droplets, Phys. Rev. Lett. 103, 164502 (2009).

[21] A. R. Thiam, N. Bremond, and J. Bibette, Breakig of an Emulsion Under ac Electric Field, Phys. Rev. Lett. 102, 188304 (2009).

[22] M. Chabert, K. D. Dorfman, and J. L. Viovy, Droplet fusion by alternating current (ac) field electrocoalescence in microchannels, Electrophoresis 26, 3706 (2005).

[23] M. Garzon, D. Adalsteinsson, L. J. Gray, and J. A. Sethian, A coupled level set-boundary integral method for moving boundary simulations, Interfaces Free Bound. 7, 277 (2005).

[24] M. Garzon, L. J. Gray, and J. A. Sethian, Numerical simulation of non-viscous liquid pinch-off using a coupled levelsetboundary integral method, J. Comput. Phys. 228, 6079 (2009).

[25] M. Garzon, L. J. Gray, and J. A. Sethian, Simulation of the droplet-to-bubble transition in a two-fluid system, Phys. Rev. E 83, 046318 (2011).

[26] M. Garzon, L. J. Gray, and J. A. Sethian, Axisymmetric boundary integral formulation for a two-fluid system, Int. J. Numer. Methods Fluids 69, 1124 (2012).

[27] A. Johansson, M. Garzon, and J. A. Sethian, A threedimensional coupled Nitsche and level set method for electrohydrodynamic potential flows in moving domains, J. Comput. Phys. 309, 88 (2016).

[28] L. J. Gray, M. Garzon, V. Mantič, and E. Graciani, Galerkin boundary integral analysis for the axisymmetric Laplace equation, Int. J. Numer. Methods Eng. 66, 2014 (2006).

[29] J. A. Sethian, Level Set Methods and Fast Marching Methods, Cambridge Monographs on Applied and Computational Mathematics (Cambridge University Press, Cambridge, 1999).

[30] D. Adalsteinsson and J. A. Sethian, The fast construction of extension velocities in level sets methods, J. Comput. Phys. 148, 2 (1999).

[31] See Supplemental Material at http://link.aps.org/supplemental/ 10.1103/PhysRevE.100.063111 for movies corresponding to the four coalescence patterns: Oscillation, satellite, repulsion and jetting. 